\title{
Development of a Refined Space Vehicle Rollout Forcing Function
}

\author{
George James ${ }^{1}$, Jon-Michael Tucker ${ }^{1}$, Gerard Valle ${ }^{1}$, Robert Grady ${ }^{1}$, John Schliesing ${ }^{1}$, \\ James Fahling ${ }^{2}$, Benjamin Emory ${ }^{3}$, Sasan Armand ${ }^{3}$ \\ ${ }^{1}$ NASA Johnson Space Center, Houston, TX 77058 \\ ${ }^{2}$ Jacobs Technology, Houston, TX 77058 \\ ${ }^{3}$ NASA Langley Research Center, Langley, VA 23681
}

For several decades, American manned spaceflight vehicles and the associated launch platforms have been transported from final assembly to the launch pad via a pr-launch phase called rollout. The rollout environment is is rich with forced harmonics and higher order effects can be used for extracting structural dynamics information. To enable this utilization, processing tools are needed to move from measured and analytical data to dynamic metrics such as transfer functions, mode shapes, modal frequencies, and damping. This paper covers the range of systems and tests that are available to estimate rollout forcing functions for the Space Launch System (SLS). The specific information covered in this paper includes: the different definitions of rollout forcing functions; the operational and developmental data sets that are available; the suite of analytical processes that are currently in-place or in-development; and the plans and future work underway to solve two immediate problems related to rollout forcing functions. Problem 1 involves estimating enforced accelerations to drive finite element models for developing design requirements for the SLS class of launch vehicles. Problem 2 involves processing rollout measured data in near real time to understand structural dynamics properties of a specific vehicle and the class to which it belongs.

\section{Nomenclature}

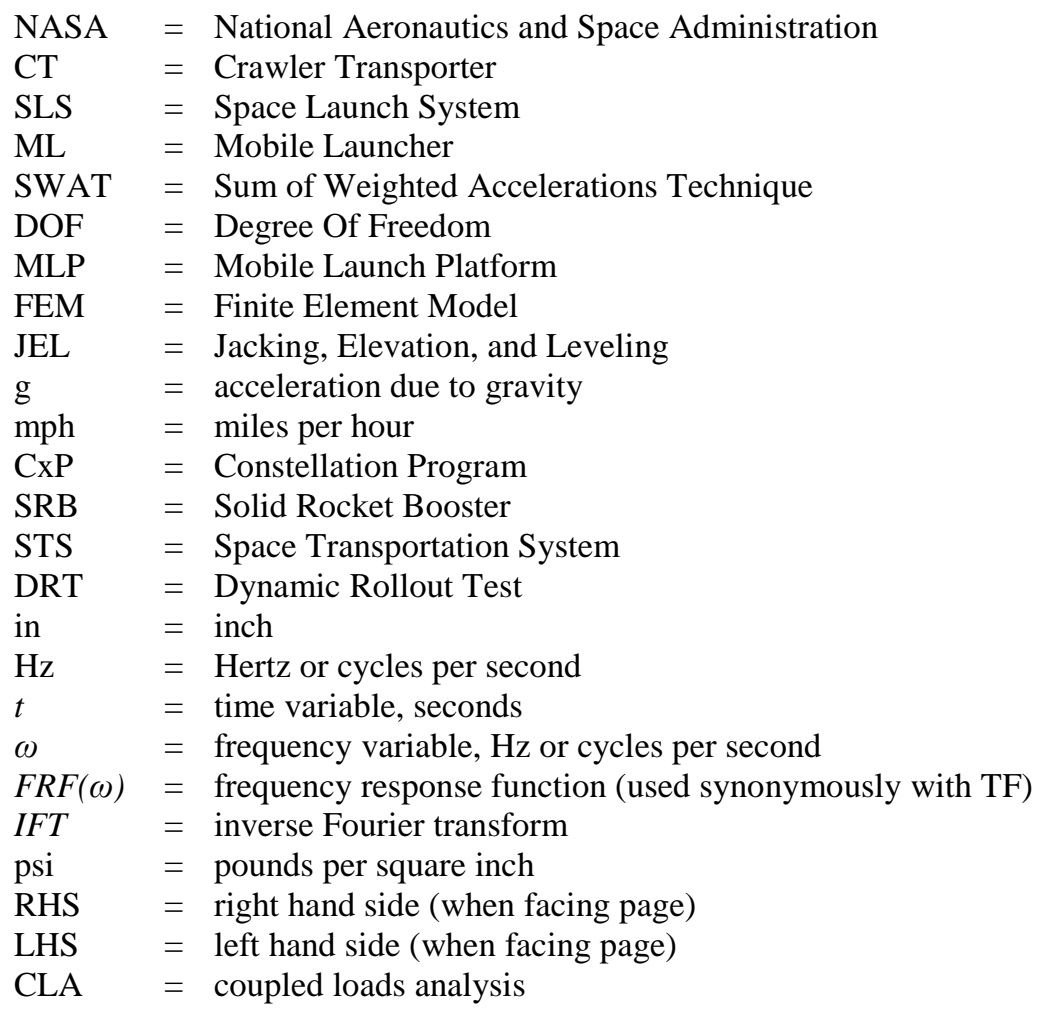




\section{Introduction}

$\mathrm{M}$ ajor NASA manned vehicles have made the final transportation to the launch pad via a significant track/tread based system called the Crawler-Transporter or CT. Figure 1 depicts the Space Launch System (SLS) vehicle and the associated Mobile Launcher (ML) under transport to the launch site using this system. This "Rollout" event has been found to produce structural and fatigue loads on large flexible launch vehicles and spacecraft. Alternatively, there is a potential to use the resultant rollout loading to exercise the vehicle for structural diagnostics and model correlation. These two situations (induced loads and operational diagnostics) are the two drivers for the current effort. Hence, this paper addresses current work to understand and reproduce the rollout forcing functions to allow refined predictions for the Space Launch System (SLS) vehicle and Mobile Launcher (ML) as well as to enable the use of the vehicle response and analytical predictions for comparisons and validation.

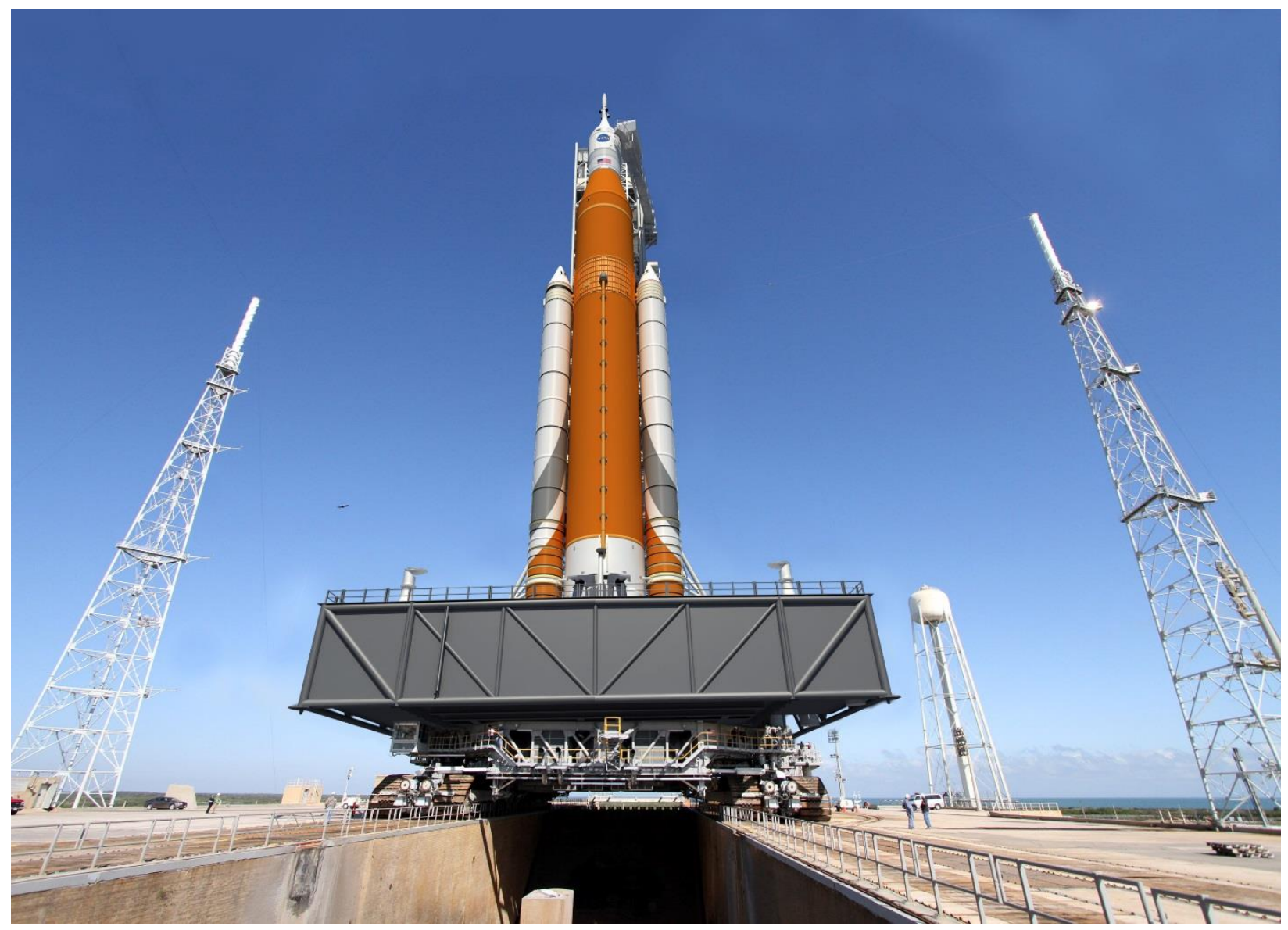

\section{Figure 1. Artist Conception of the Space Launch System (SLS) and Mobile Launcher (ML) on the Crawler-Transporter (CT) (from http://www.nasa.gov)}

Rollout forcing functions are generated as the CT imposes a series of harmonic excitations (sine and cosine-like waveforms) onto the entire system under transport. Previous work during rollout of the Space Shuttle system found two primary families of harmonics, which are characterized by a speed-dependent harmonic load and integer frequency superharmonics ${ }^{1,4}$. This loading is inherent in the tracked vehicle design of the CT and the frequency content is directly tied to the speed of the CT. Vehicle response can significantly increase when one of these harmonic forcing function harmonics is at or near one of the resonant frequencies of the vehicle. Figure 2 shows a Campbell diagram (harmonic frequency content vs. rollout speed) showing the possible interactions between the forcing function harmonics and the vehicle models of the Space Shuttle vehicle. The two families of harmonics are listed as the .59 family and the .975 family. Figure 3 shows one of the eight CT Tracked drive systems with the hardware dimensions 
that are related to these two harmonic families of the forcing functions. The .98 family is related to the 18 inch spacing between each of the 57 shoes in a track. The .59 famility is related to the 30 inch distance between the rollers that support the entire system and allow it transverse across the tracks.
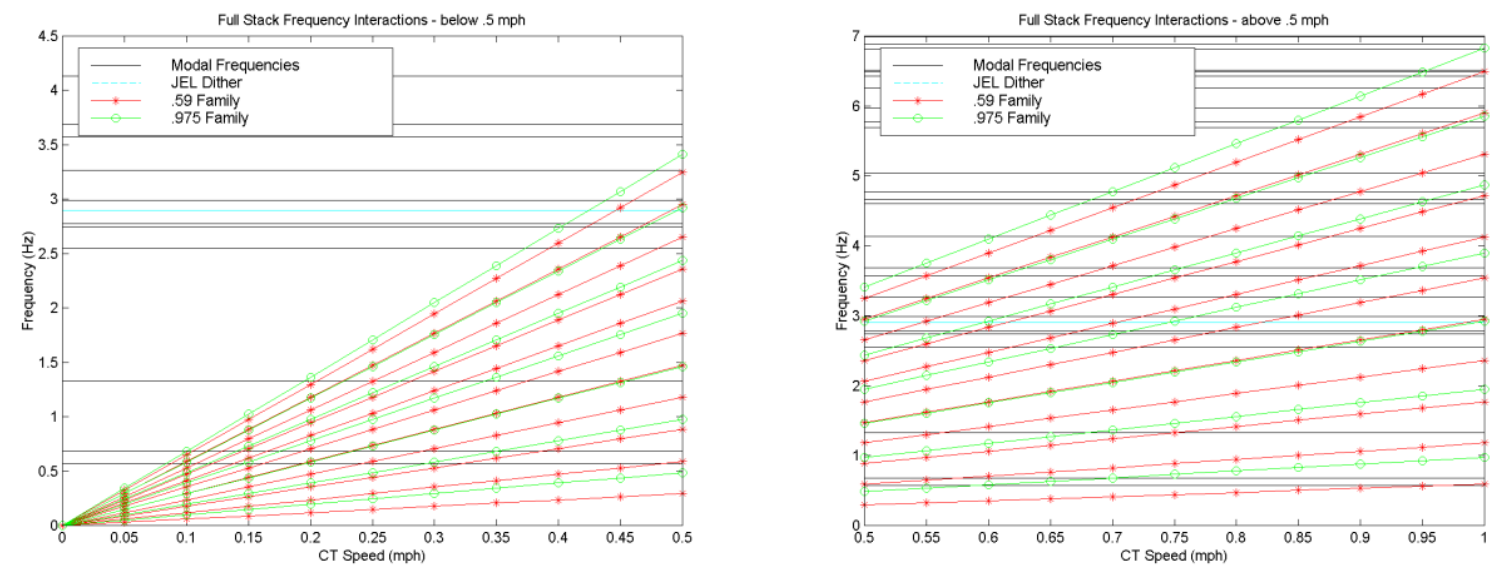

Figure 2. Harmonic Frequencies of the Space Shuttle era Rollout Forcing Functions vs. CT Speed

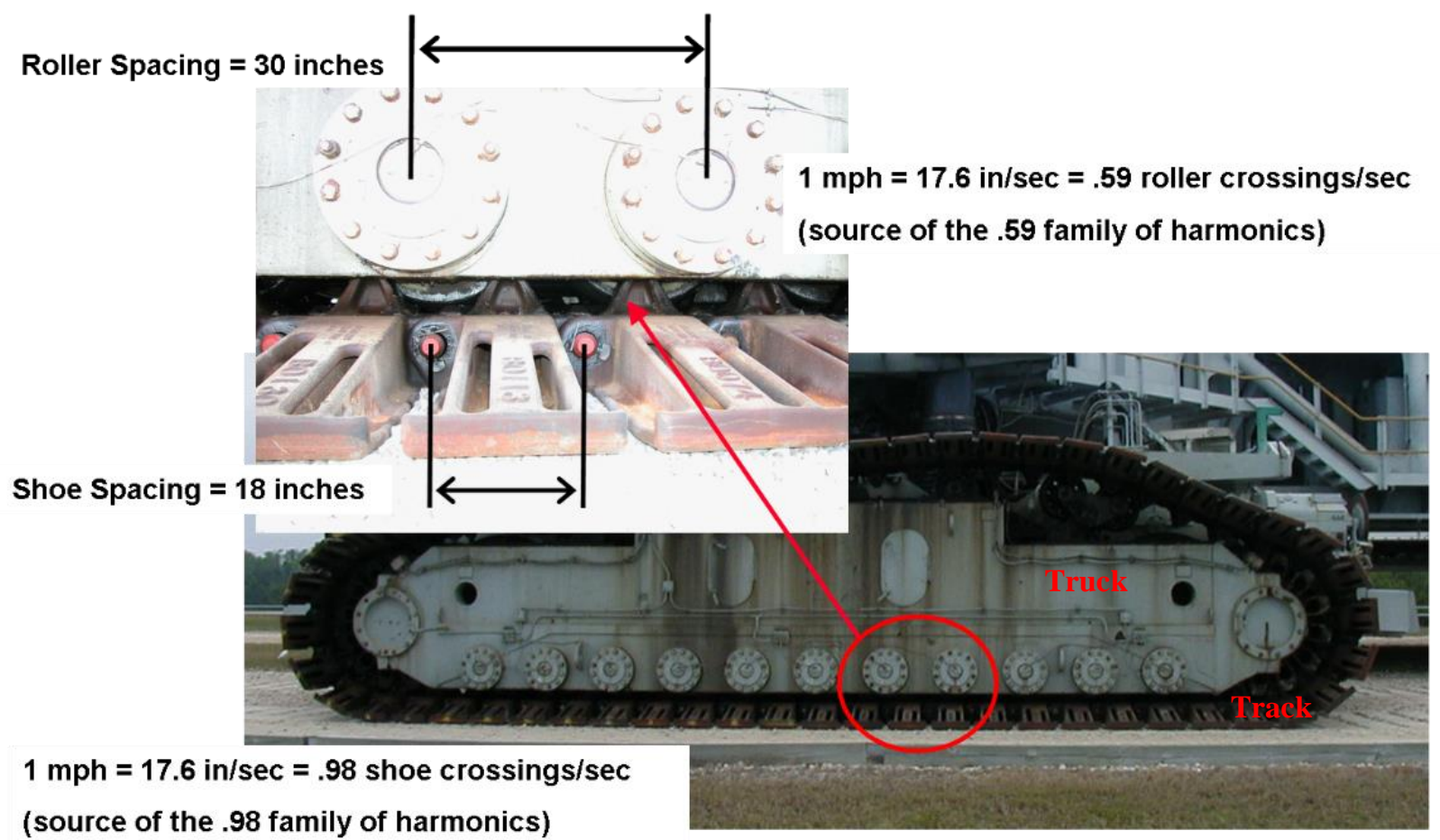

Figure 3. Enforced Displacement Estimate based on the Roller Track Contact Model

In previous Shuttle-era work on the rollout forcing functions, the primary effort was to avoid speed ranges that caused undesired loading events ${ }^{1,4-8}$. Hence, this historical work has formed a useful basis for initiating the current effort but was not driven by the same high-fidelity need to understand the forcing functions as the current work. Previously, the forcing functions were estimated using a modification of the Sandia National Laboratories-developed Sum of Weighted Accelerations Technique (SWAT) ${ }^{1,2,3}$. The modifications to SWAT resulted in a hybrid approach that used the mode shapes of the structural model to estimate weighting parameters, which operated on measured accelerations. Estimated six Degrees-Of-Freedom (DOF) forces then drove a free-free model of the Shuttle vehicle and Mobile Launch Platform (MLP) at the center-of-mass. The actual CT was not modelled in this work. The resulting functions were then adjusted to simulate unmeasured roll-out speeds. This process allowed the dynamic model of the structure

American Institute of Aeronautics and Astronautics 
to be used to estimate the response at a wider range of speeds than the measured data-base. The problems with this approach are the reliance on a center-of-mass input point, a limited frequency range constrained by the number of measurement locations, the reliance on the analytical Finite Element Model (FEM) of the vehicle, and the lack of direct CT data in the process. Post-Shuttle work with the Ares I-X vehicle highlighted the potential uses of the expanded Shuttle-era data to estimate rollout forcing functions ${ }^{9}$. More recently, the need for a high-fidelity understanding of the rollout environment to service the SLS needs requires that a forcing function estimation process be developed which reduces the impact on these historical shortcomings and builds on the most recent work to understand and model this phenomena ${ }^{10}$. This paper covers the efforts underway to develop such a higher fidelity understanding of the rollout forcing functions in order to estimate induced loads and perform operational diagnostics.

\section{Description of Rollout Hardware}

There are multiple definitions for the term "forcing functions". However, in order to understand these different definitions, it is necessary to define the different hardware components of the CT and Launch Platforms. The insert of Figure 3 shows four of the 57 shoes that make up the track in contact with the ground. Two of the 11 rollers per track are barely visible but the locations are clearly understood based on the circular access covers. The primary image of Figure 3 shows the signcant structural member (called the truck) that holds a pair of tracks with 11 rollers per side of each truck. Each of the four trucks has a Jacking, Elevation, and Levelling (JEL) and a guide tube system to properly support the CT chassis and the payload (launch platform and launch vehicle) as well as four electric track motors to provide motive force. The guide tube transfers all lateral loads from the CT chassis, the launch platform, and the launch vehicle into each of the four trucks. Figure 4 shows a schematic of a CT truck with the tracks, track motors, and guide tube marked.

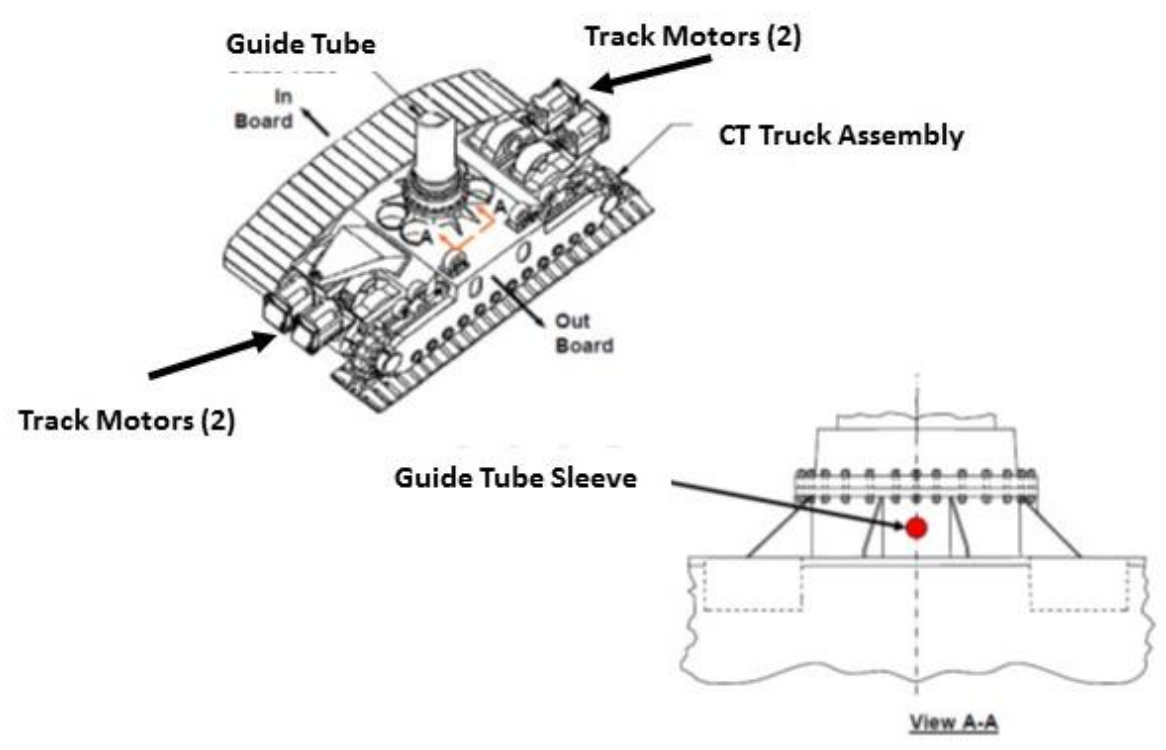

Figure 4. Schematic of CT Truck Showing the Guide Tube to Support Lateral Loads

All axial forces that are generated in the process of enableing the CT trucks to support the CT chassis, launch platform, and launch vehicle are carried via the JEL systems. Each truck has four hydraulic cylinders that compromise the JEL system. The variability of the JEL system allows the CT to carry variable weight payloads, to handle uneven weight dispersions, to lift and lower the payload, and to level the payload even when moving up or down the ramp at the pad. The CT chassis provides the structural framework to connect and control/steer all four trucks as well as interface to the launch platform. Also the chassis contains the diesel motors that drive the generators for motive and auxiliary power. The chassis contains the hydraulic systems for the JEL and steering functions. And finally all control interfaces to allow operation of the CT are housed in the chassis. Figure 5 shows a schematic and a photo showing 
the location and configuration of the JEL system on each truck, as well as the CT chassis. Also note that one of the four Launch Platform support points is denoted in Figure 5. There is a pickup point for the launch platform at the center of each JEL cylinder set on each truck.
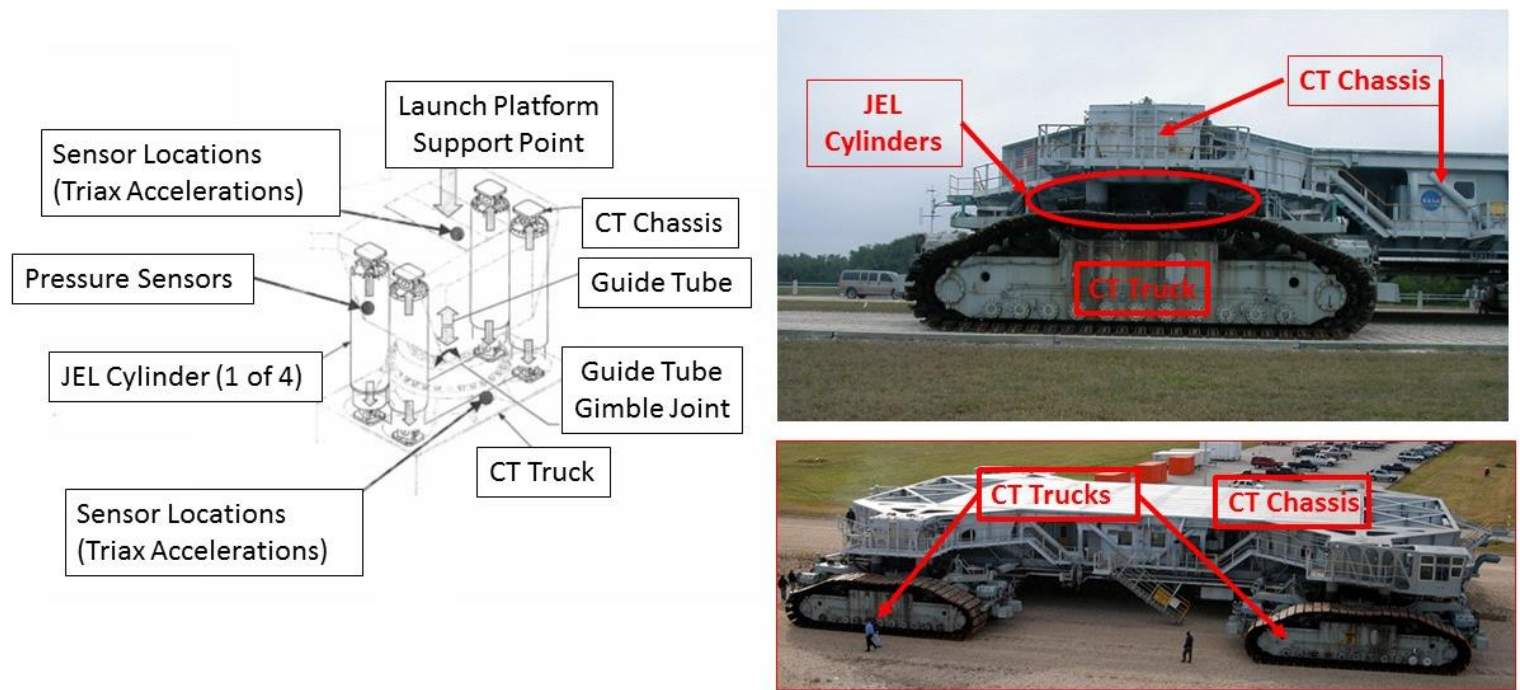

Figure 5. Schematic and Photos of CT Jacking, Elevation, and Leveling (JEL) Systems and Chassis

There have been three launch platforms that have developed for four primary payloads (by weight) for the CT during the scope of the work described herein. Additionally, there were earlier launch platforms for the Apollo/Saturn launch vehicles but data from those programs has not been carried forward into the current efforts. The Space Shuttle and the Ares $1-\mathrm{X}^{9}$ vehicles used the Shuttle Mobile launch Platform (MLP). This platform weighed 8.2 million pounds and is easily recognized due to the lack of a launch tower ${ }^{11}$. There was a 9.5 million pound launch platform built specifically for the cancelled Constellation Program (CxP) that was called a Mobile Launcher (ML) ${ }^{12}$. This platform will be referred to as the CxP ML. The CxP ML was rebuilt to generate the ML for the Space Launch System (SLS) vehicle. This 10.5 million pound platform will be referred to as the SLS ML ${ }^{13}$. Figure 6 shows each of these platforms during transport by the CT. Note that the SLS ML is a conceptual image as an SLS vehicle has not been mounted on the SLS ML as of this writing.

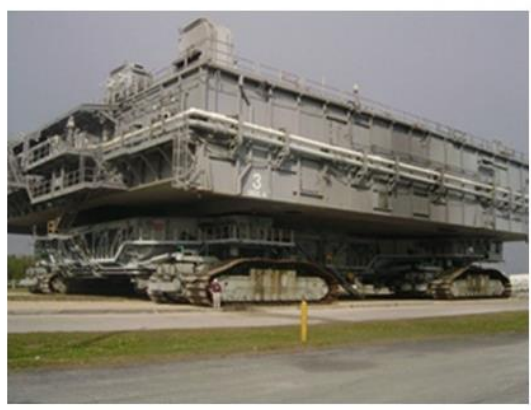

Shuttle MLP

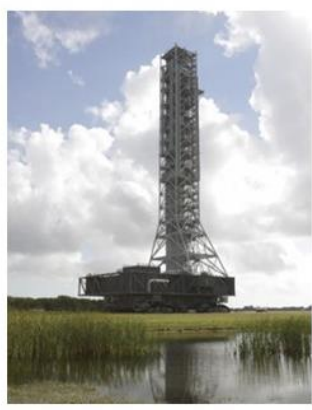

CXP ML

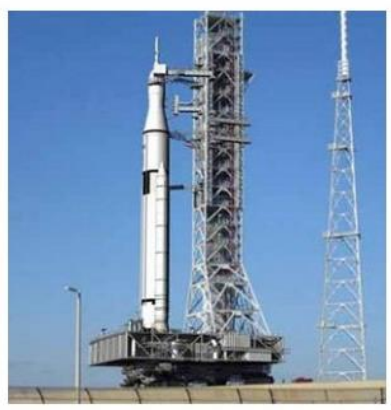

SLS and ML

Figure 6. Space Shuttle, Constellation, and Space Launch System Launch Platforms

\section{Definitions of Rollout Forcing Functions}

The next critical information needed for this work is to describe the different ways to define and provide "forcing functions". Historically multiple definitions have been used to define rollout forcing functions and the defined products of the current work continue that trend. Fundamentally the current level of understanding actually requires

American Institute of Aeronautics and Astronautics 


\section{$11 / 21 / 16$}

that the rollout forcing functions be assessed from multiple vantage points (hence using different definitions) to overcome the lack of complete data sets. This section covers these different definitions or vantage points).

\section{Input Forces}

The most obvious definition of "forcing functions" are input forces (units of pounds force or Newtons) that drive the system. With the systems under consideration here, these inputs may be defined at any of several interfaces: the forward motive force provided by each CT truck; the ground/shoe interface at each track; the truck roller to track shoe interface; the gross truck force inputs to the JEL and guide tube systems, the actual forces generated by each truck's JEL and guide tube systems, the forces acting at the four CT to launch platform interfaces on the chassis, or the various interfaces between the launch platform and launch vehicle. Each of these different input force defintions have different advantages and disadvantages and require synchronizing the definition of the system of interest as well. As the interface used for force definition moves away from the control inputs and toward the actual launch vehicle, more of the system has to be used in defining the force and less is used to define the system. In general, it becomes harder to instrument or estimate the more basic low level forces very close to the control or ground inputs but it becomes harder to interprete the forces transmitted through more structure. As will be discussed later, the current efforts are enhanced by the fact that models of the CT are now available.

The historical Shuttle work performed previously was primarily focused on estimating forces at the CT/MLP interface (using the center-of-mass as a surrogate input point) ${ }^{1}$ or the MLP/Launch Vehicle interface ${ }^{7}$; although there were limited efforts to utilize forces generated by the JEL systems ${ }^{5}$. Input forces were chosen for the original Shuttle work to allow inputs to the models to be defined in such a manner as to allow the data to be shifted to estimate vehicle response at rollout speeds that were not measured. In this way, a rollout speed could be chosen that reduced the fatigue loads on the flight vehicle ${ }^{1}$. From this experience, it is clear that the primary advantage of the input force definition is that the resulting forces are basic quantities (i.e. less affected bystructural dynamics), which can potentially be modified for unmeasured systems or operating enviornments. However, the disadvantage is that measurement or estimation of the forces are more difficult than other assumed forcing function definitions.

\section{Input Accelerations}

The most common approach to define rollout forcing functions are as input accelerations (units of meters per second squared, inches per second squared, or the non-dimensional units of g's) $)^{9}$. The Shuttle rollout work before 2000 was all based on driving a model of the Shuttle vehicle and MLP with measured accelerations from operational rollouts. This operational reliance on measured acclerations driving such a system continued to the end of the Shuttle Program ${ }^{8}$. As a result of the operational and developmental efforts, there is a significant Shuttle-era database of input accelerations available. This database can enable acceleration inputs to be defined at any of three interfaces: the inputs to the JEL system, the inputs to the launch platform, and the inputs to the vehicle. In fact, this acceleration data is continuing to define the rollout loads and rollout operations for the SLS system. The Shuttle, Ares1-X, and SLS vehicles analyses have used this acceleration database at the CT/launch platform interface to estimate rollout loads.

The advantage of using input accelerations is that measurements can easily be obtained using accelerometers at the desired interfaces. The disadvantage is that those measurements become strongly tied to the actual system and operating conditions under test due to sensitivity to the payload structural dynamics. One of the primary tasks of the current effort is to develop CT/MLP interface acclerations that are appropriate for SLS-era systems (CT, ML, and launch vehicle) such that the current Shuttle-era input acceleration database can be assessed for conservatism. For this work, analyses and data at other forcing function interfaces will need to be used to handle dynamic sensitivities.

\section{Input Displacements}

An alternative definition of the forcing function that is currently being studied are displacements. A displacement forcing function only makes sense for low motion areas of the structure that are likely to be unaffected by structural dynamics such as at the shoe/ground interface of the roller/shoe interface. Displacements are not be as easy to measure as accelerations but are proving to be more stable and easier to estimate than forces. However, there are technical challenges to overcome in developing appropriate displacement forcing functions at the base interfaces meantioned earlier. One driver of these issues are the large number of input locations to be covered. There are 11 rollers per track and two tracks per truck and 4 trucks on the CT for a total of 88 rollers on the system. If three DOFs per roller are assumed, then there are 264 displacement time histories needed to drive the system. 


\section{Indirect Measurements}

Another alternate definition of forcing function is that of indirect measurements. These would be measurements that are indirectly related to input foces. The examples include electrical currents feeding propulsion motors, tachnometer outputs from propulsion motors, JEL system pressures, guide tube strains and hold down post strains at the launch vehicle interface. Past work has been performed to process hold down post strains to directly drive a model of the launch vehicle ${ }^{7}$ or as a supplemental input to other measurements ${ }^{14}$. These indirect meausrements are proving useful to supplement other incomplete measurements.

\section{Measured Data Available for Rollout Forcing Functions}

The second critical input needed to provide rollout forcing functions for the two products mentioned earlier are to understand what measured data sets are available. This section provides an overview of the data that is available for this work.

\section{CT-Only(Shuttle)}

A series of instrumented rolls of the CT were performed in 2005 . The system was operated at .5, .6., .7, .75, .8, and .9 miles per hour. There was also a data take covering a speed sweep of of this same speed range. The CT was instrumented with triaxial accelerometers mounted at 9 locations, JEL system pressure transducers, and a tachometer speed sensor on one of the 16 propulsion motors. The configuration is the same as that seen in Figure 5.

\section{CT-Only(SLS)}

One of the CTs was structurally modified to handle the larger weight of the SLS stack and ML. As of this writing there are discussions underway to perform a series of dedicated tests in 2017 at several speeds and with a similar sensor set to the Shuttle-era tests. There are also discussions underway to add guide tube strains to the sensor suite, which would allow an estimate of lateral forces between the CT trucks and the CT chassis. The configuration would also be similar that seen in Figure 5.

\section{MLP+CT(Shuttle)}

The CT loaded with an empty MLP was also subjected to a series of rollout tests in 2005. The system was operated at $0.0, .5, .6 ., .7, .8$, and .9 miles per hour. There were $9 \mathrm{CT}$ triaxial accelerometer locations, 12 MLP triaxial accelerometer locations, JEL system pressure transducers, wind speed, wind direction, and a tachometer speed sensor on one of the 16 propulsion motors. The configuration is the same as that seen in Figure 6.

\section{MLP+CT(SLS)}

There are currently discussions to perform rollout testing with the upgraded CT loaded with an empty MLP in 2017. The system would be operated at multiple speeds. The CT would be instrumented similar to the 2005 test but the MLP would be unistrumented. The configuration is the same as that seen in Figure 6.

\section{$\operatorname{ML}(\mathbf{C x P})+\mathrm{CT}($ Shuttle)}

In 2011, there was an instrumented roll with the Constellation (CxP) ML. This ML was similar to the current SLS version of the ML with a significant launch tower. The CT was instrumented as was the previous Shuttle-era tests with additional instruments on the ML. This is an important data set as it is transitional between the Shuttle and SLS generations of hardware. An image of the test is shown in Figure 7. Another interesting aspect of this test is that some of the highest instrumented rollout speeds were captured during this test. Efforts are currently underway to recover this data from archival sources.

\section{ML(SLS)+CT(SLS)}

There are plans to roll the current SLS ML to the pad using the upgraded SLS version of the CT in 2017. Discussions and planning are underway to instrument the CT and the ML during this activity. Furthermore, discussions are underway to assess the ability to roll at different speeds for dedicated data takes. The configuration would be the SLS ML with tower as in the righthand image of Figure 6 but without the SLS launch vehicle.

\section{Ares I-X+MLP+CT(Shuttle)}

The AresI-X vehicle rollout to the pad in 2009 was instrumented with CT accels and four accelerometers on the MLP and a single triaxial accelerometer set on the vehicle. There were also four strain measurements at the MLP to vehicle interface (one per hold-down post). The rollout configuration is seen in Figure 7. 


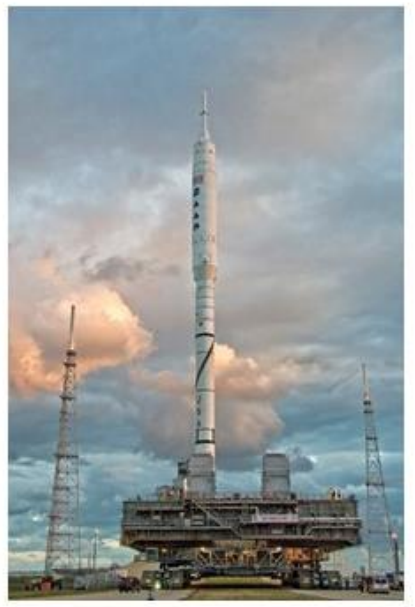

Ares 1-X

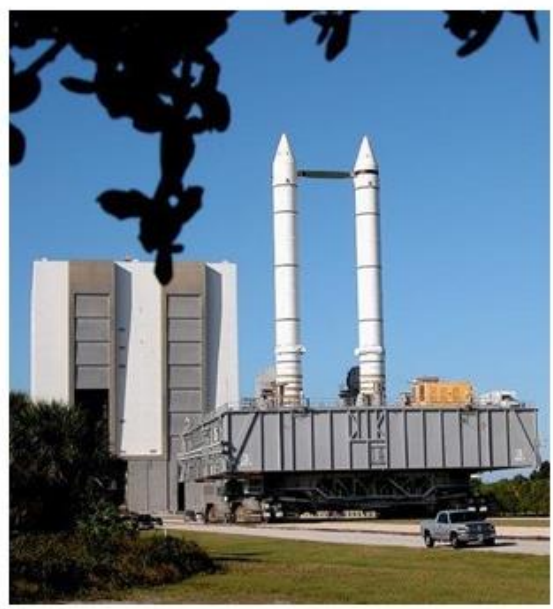

Shuttle Partial Stack

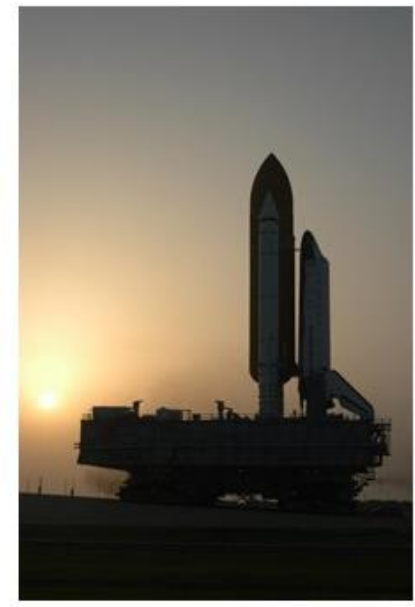

STS-115

\section{Figure 7. Ares I-X, Shuttle Partial Stack, and Shuttle STS-115 Rollouts}

\section{Shuttle Partial Stack+ MLP+CT(Shuttle)}

The Shuttle partial stack consisted of a pair of Solid Rocket Boosters (SRBs) and a connecting crossbeam that was a major structural component of the External Tank. This configuration was used for a significant round of rollout testing in 2003. The CT sensor suite mentioned previously was used as well as 14 triaxial accelerometer packages on the MLP and three triaxial accelerometer locations on each SRB. There were also four strain measurements at each MLP to SRB interface (one per hold-down post) as well as diagnostic video imagery. This test series contained the most detailed range of speeds recorded to-date. This configuration is seen in the middle of Figure 7.

\section{STS-115+MLP+CT(Shuttle)}

The Shuttle stack configured for the STS-115 launch was instrumented to acquire data for verifying the rollout fatigue loads and operational speeds. Data from several speeds were obtained during the roll to the pad. The standard suite of CT accels, pressures, and speed were acquired. Nine accelerometers were mounted on each SRB. The body of the MLP had 12 triax accelerometer locations with an additional eight triax locations at the MLP/SRB interfaces. The Orbiter had 13 triax accelerometer locations on the structure plus another 10 triax locations on the Space Shuttle Main Engines (SSMEs). There were additional strain measurements on the vehicle as well. The STS-115 configuration is seen in Figure 7. It should be noted that several additional Shuttle rollouts were also instrumented with a more limited set of sensors but none have provided the utility of the STS-115 test.

\section{SLS+ML(SLS)+CT(SLS)}

One of the primary targets for the work reported in this paper is to support an instrumented roll to the pad with the full STS stack in 2018. This test has been named the Dynamic Rollout Test or DRT. The configuration is represented in Figures 1 and 6. A significant instrumentation suite and multiple speeds are under discussion for implementation. The DRT is unique in that it has the potential to partially mitigate the inability to test the entire launch vehicle in a traditional modal test.

\section{Analytical Data Available for Rollout Forcing Functions}

A third critical input to this work is an understanding of the analytical data that is (or can expected to be) available. This section provides an overview of the different analytical data classes that are being used to consolidate the different definitions of "forcing function" and combine the different measured data sets to allow (1) assessment (and delivery if necessary) of current rollout forcing functions and (2) development of a process to analyze DRT rollout data for correlation and diagnostics. 


\section{Structural Dynamic Finite Element Models}

The ability to perform modal and transient structural dynamics analysis via Finite Element Models (FEMs) is one of the most useful analytical tools to support the development and assessment of forcing functions. Currently FEM models exist for all of the major components (launch vehicle, launch platform, and CT) discussed in the previous section except for the SLS version of the CT. However, several models need to be integrated to form the proper launch stack and some of the critical models still need to be validated. In fact, validation of the SLS versions of all three component FEMs is one of the primary driving requirements behind this work. As an example, Figure 8 shows the mesh of the model of the Shuttle partial stack on an MLP with the model of the CT chassis. Figure 7 shows the actual structure that is represented by the FEM.
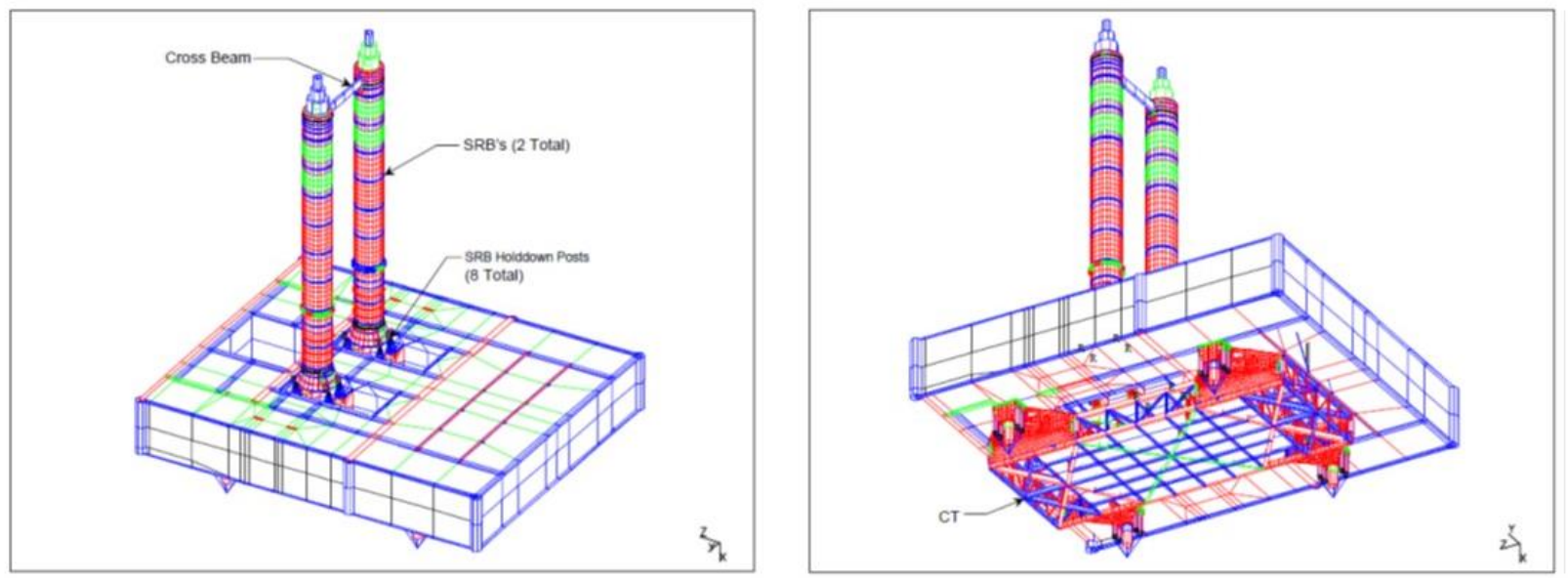

Figure 8. Shuttle Partial Stack Finite Element Model with MLP and CT Chassis

It should also be noted that the FEM representation can be necessary to enable other analytical results - as will be seen in the next set of analytical data (SWAT-estimated forces). However it has previously been mentioned that directly driving an FEM transient result with measured accelerations is the most commonly used manifestation of rollout forcing functions ${ }^{7,9}$. The first required product of this effort is to combine available measured data and analytical data to validate input acceleration time histories to drive and FEM of a system that has not yet been tested. One of the approaches to develop the necessary rools and understanding is to drive and FEM of the CT with accelerations measured as close to the interface as possible. The complications of this part of the work are that the FEM of the CT has not been fully validated and that stiffness between the ground/shoe/roller interfaces must still be estimated. Figure 9 shows an example of this work (top plot - time domain and bottom plot - frequency domain) using data and FEM of the CT only while moving at $.7 \mathrm{mph}$. This work is an early iteration that is being used to set the spring to ground stiffness for three DOF at all 88 rollers. The noise in the results and the forced harmonic peaks are captured while an analytical offset is a indicative of the ongoing efforts to set the ground spring stiffnesses.

\section{Platform/Vehicle C.G. Force Estimates}

Previous Shuttle work estimated forces at the CT/MLP interface using data from the Partial Stack and STS-115 tests ${ }^{1}$. These forces were estimated using a modification of the Sandia National Laboratories Sum of Weighted Accelerations Technique (SWAT) ${ }^{2,3}$. In this technique, the forces at the Centrer of Gravity (C.G.) are estimated using a weighted summation of measured acceleration as shown in equation (1).

$$
F_{C G_{i}}=\underline{\phi}_{i}^{T} \underline{f}(t)=m_{i} a_{C G_{i}}=m_{i} \underline{w}_{i}^{T} \underline{a}(t) ;
$$

where $\mathrm{F}_{\mathrm{CGi}}$ are a matrix of the six time histories of forces and moments defined at the C.G.,

$\underline{a}(t)$ are a matrix of time histories of measured accelerations,

$m_{i}$ are the rigid body mass properties defined at the C.G., and

$\underline{w}^{T} i$ are a matrix of calculated weighting parameters.

The weighting parameters are chosen to sum the measured accelerations to generate estimates for each of the six rigid body accelerations of the C.G. In performing this weighting, the chosen elastic body generalized DOFs and all rigid

American Institute of Aeronautics and Astronautics 
body generalized modes (except the single rigid body mode of interest) are zeroed out. This statement is effectively implemented by choosing the weight matrix to vector multiply the elastic mode shapes to produce zero while unit normalized via the rigid body shapes as depicted in equations (2) and (3).

where $\phi$ are elastic mode shapes.

$$
\left[\underline{w}^{T} * \underline{\phi}\right]=[0]
$$

$$
\underline{w}_{i}^{T} * \underline{\phi}^{R}{ }_{i k}=\delta_{i k}
$$

where $\delta_{i k}$ is the delta function which is only a non-zero value (1.0) if $i=k$, and

$\phi^{R}$ are rigid body mode shapes.

Note that references ${ }^{1-3}$ contain more details on this approach.

There are multiple approaches to calculate the weight matrix from meansured data provided by the developers of the approach. However, for the previous Shuttle work, the SWAT approach had to be modified to use elastic mode shapes provided by the FEM of the system of interest. This experimental/analytical hybrid approach was necessary as it was not feasible to modal test the entire Shuttle rollout stack. The resulting forces were less affected by the presence of the elastic modes of the system when compared to the original meansured accelerations. As a result, the hybrid forces could be shifted approximate the forces expected form unmeansred rollout speeds. Figure 10 shows a result form this work that included the estimated forces at a $1.00 \mathrm{mph}$ rollout speed (top and bottom plots) and a forces predicted at a $1.04 \mathrm{mph}$ rollout speed (middle and bottom plots). Although this approach was not generally applicable for more general analyses, the results showed the utility of choosing forcing functions with less sensitivity to the structural dynamics of the system.
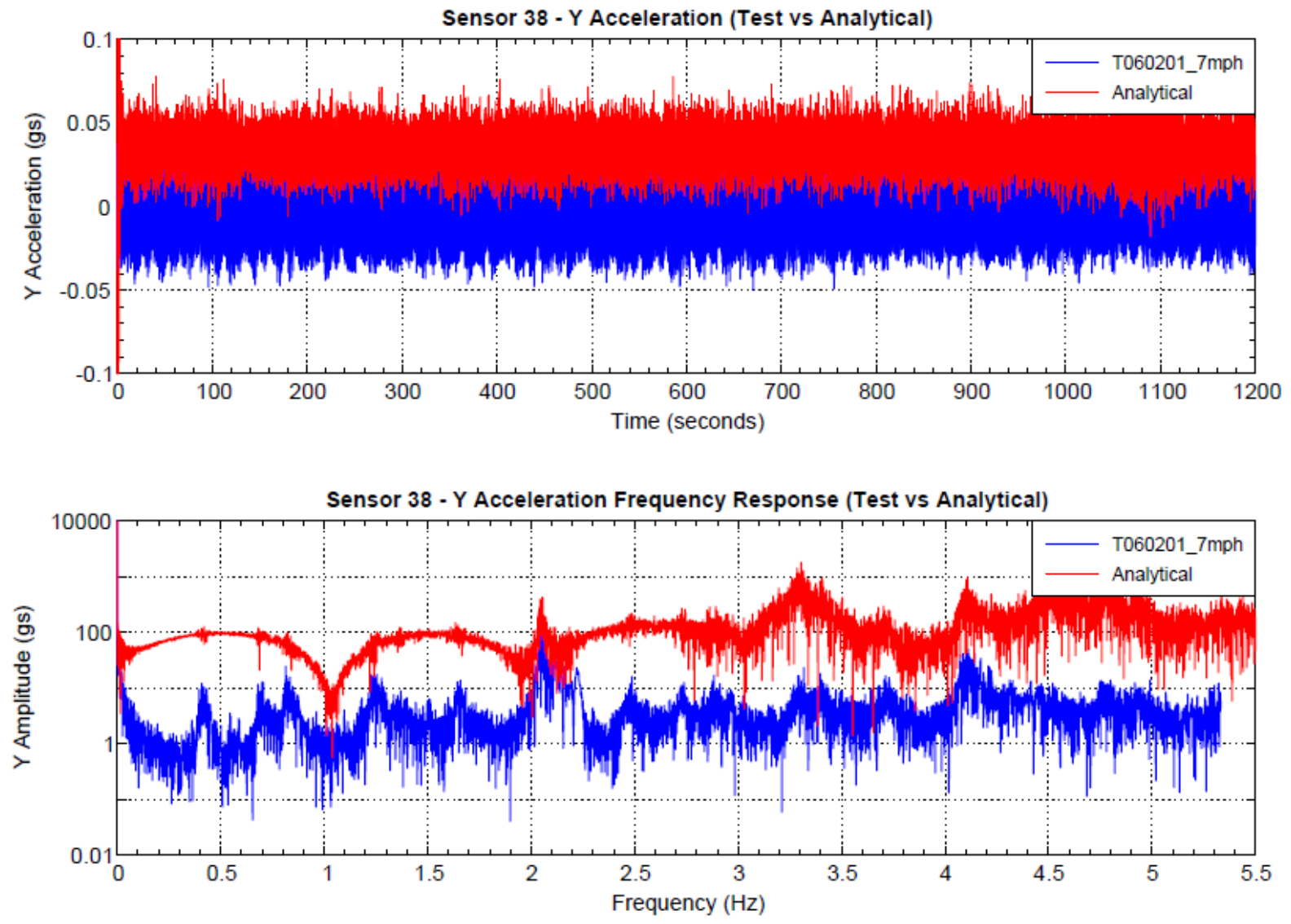

Figure 9. Enforced Acceleration FEM Solution in Vertical Direction at Center of CT Chassis

American Institute of Aeronautics and Astronautics 

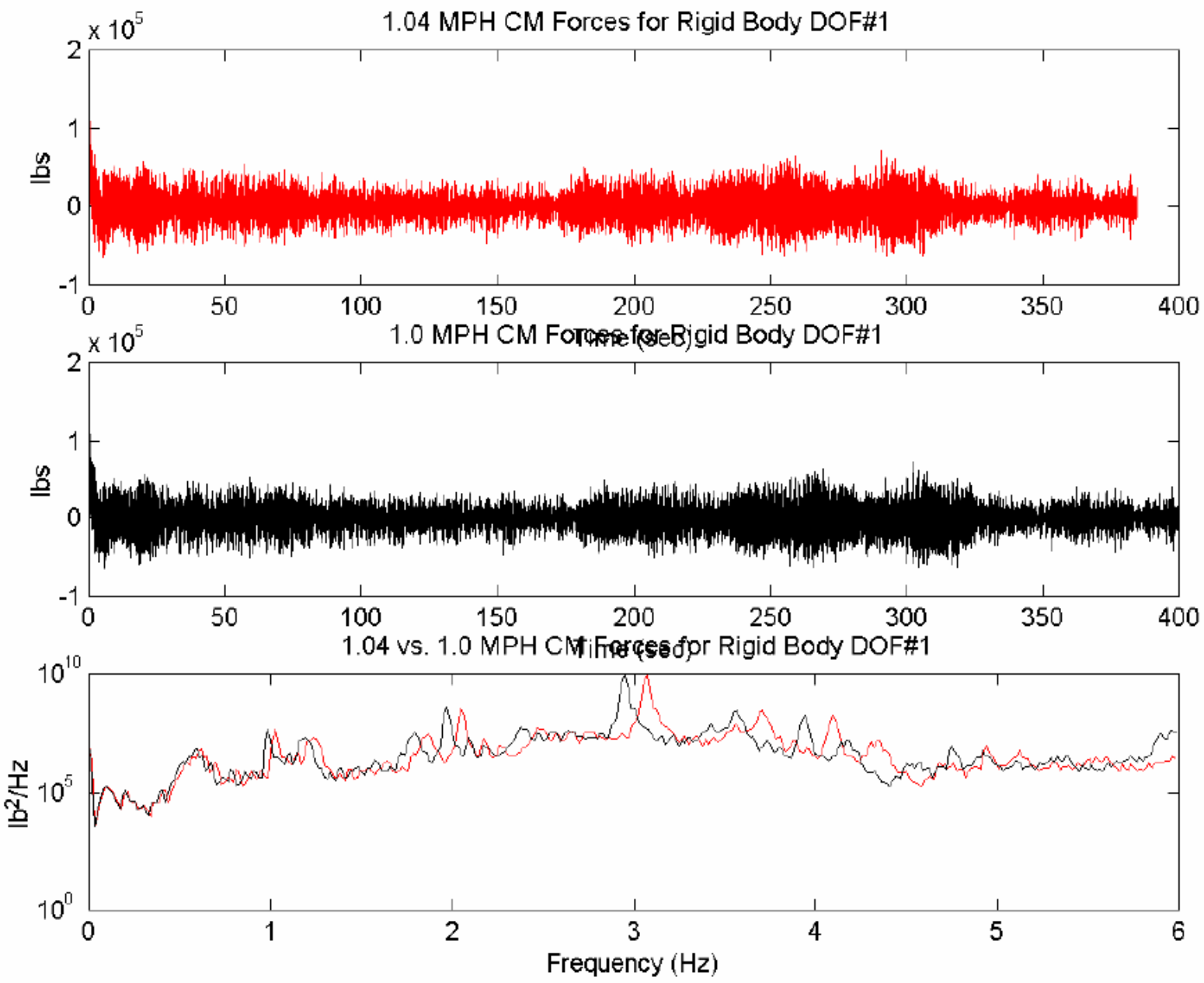

Figure 10. Estimated Forces at Center-of-Mass of STS/MLP Stack for Two Speeds

\section{Synthetic Displacement Forcing Function}

Another analytical dataset for developing rollout forcing functions are assumed displacements of the roller axles as moving along the tracks and crossing shoes. Assumptions for the roller displacements were derived from knowledge of the hardware, assessments of response measurements, inputs and results from other analytical studies ${ }^{10}$, additional measurements roller/shoe/ground interactions ${ }^{15}$, and assumed relationships between the different displacement directions/rollers. Figure 11 shows and an example of an assumed vetical displacement forcing function using a contact model derived from hardware observations. Figure 12 provides an assumed vertical displacement based on an assumption all rollers on a truck are rigidly connected and responding to rigid body motions. In Figures 11 and 12 , the top plot contains the complete 1200 second assumed time history of the vertical direction of a lead roller as it moves across the track at $.7 \mathrm{mph}$. The middle plot shows an expanded view of only a few seconds of data. The bottom plot shows the same data in the frequency domain. It can be seen in Figure 11 that only the shoe spacing family of harmonics are present (peaks separated by .7 Hz) as each roller is assumed to act independently. The roller spacing family harmonics (peaks separated by $.3 \mathrm{~Hz}$ ) are seen in Figure 12 as the rollers rigidly interact with each other. However, the magnitudes of the roller spacing harmonics are significantly suppressed. These synthetic forcing functions are assessed by driving an FEM of the individual truck or the complete CT. Comparisons to measured data allow sensitivity studies and refinements to be performed. In general this analysis approach proved useful for developing basic understanding and limiting the search space for follow-on techniques. The lesson learned was that the shoe spacing family of harmonics were directly produced. However, the roller spacing family of harmonics were an indirect emergent effect. Hence, more refined analysis and data-derived approaches were found to be required to produce forcing functions that capture the known physics.

American Institute of Aeronautics and Astronautics 

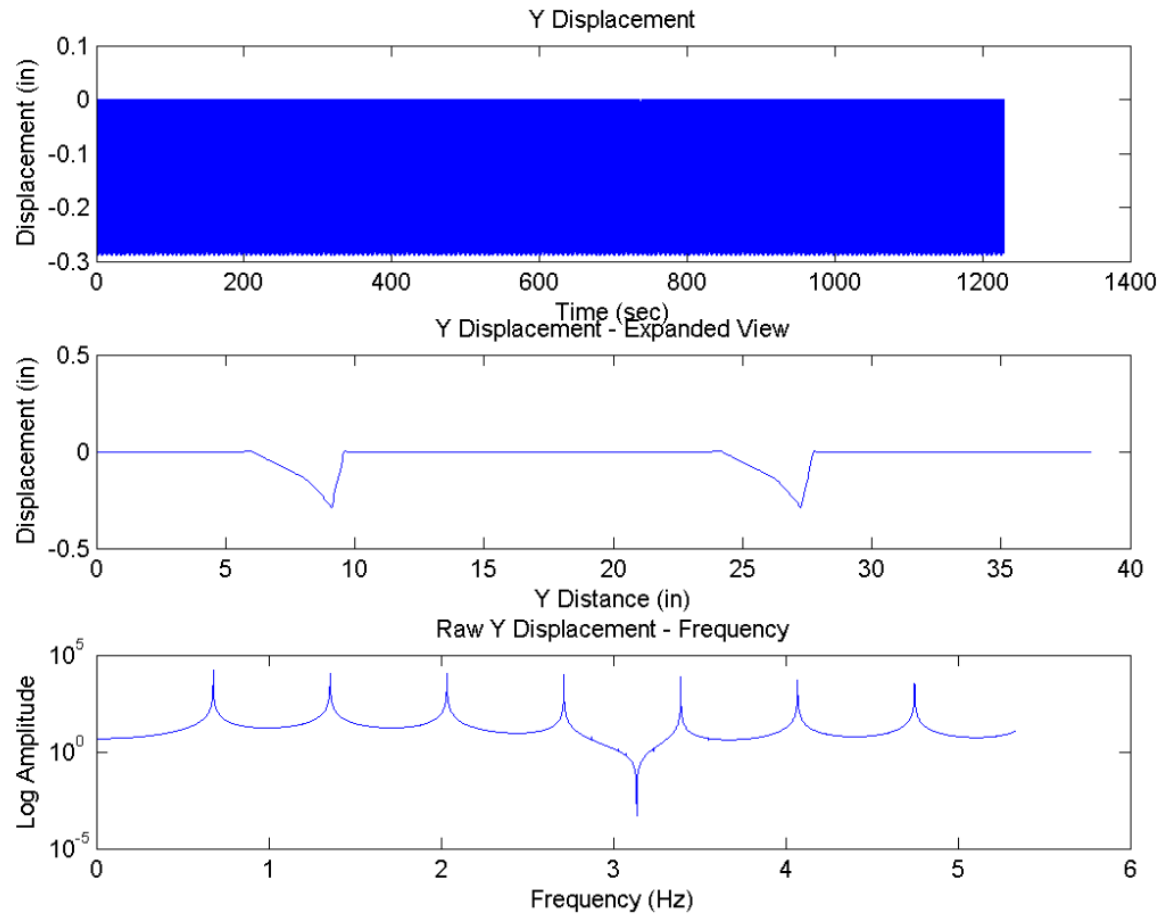

Figure 11. Synthetic Enforced Displacement Estimate Based on a Roller Track Contact Model
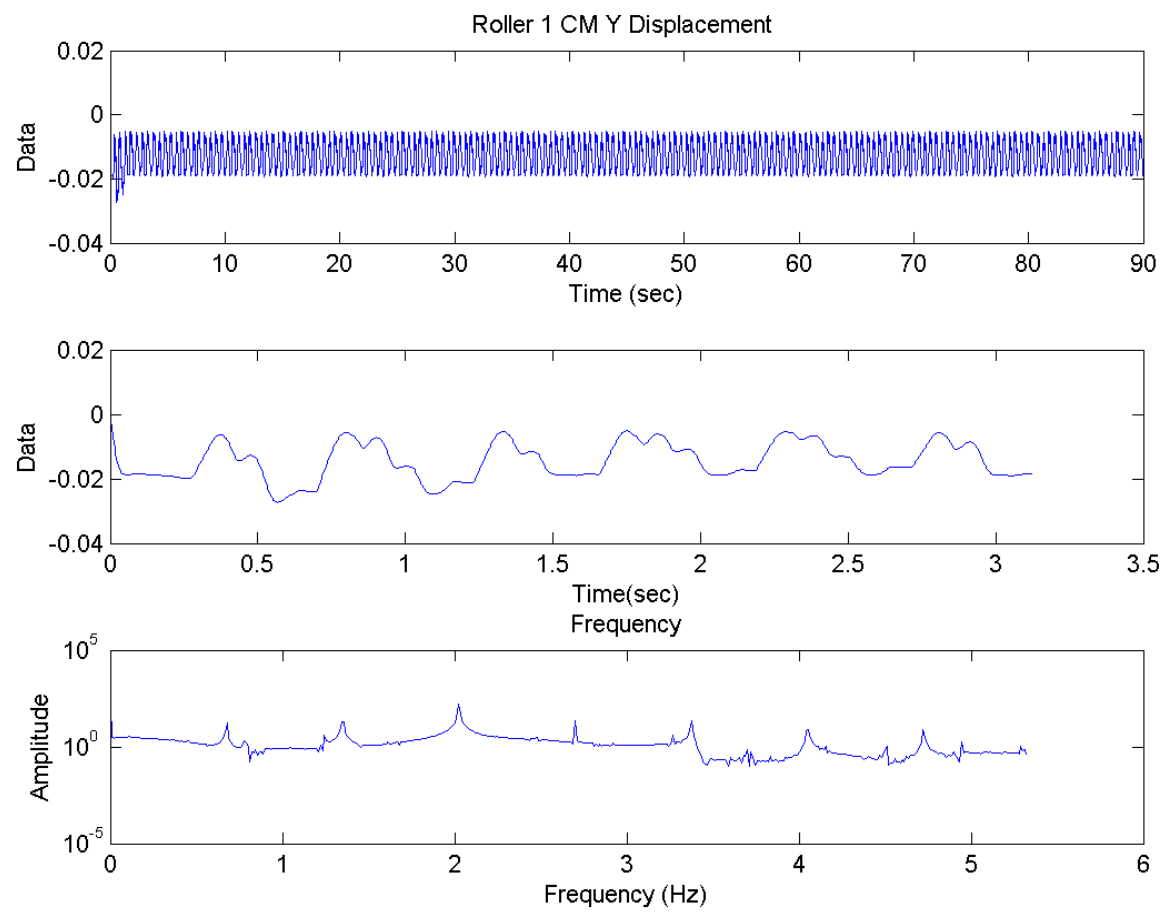

Figure 12. Synthetic Enforced Displacement Estimate Based on 11 Rigidly-Connected Rollers 


\section{$11 / 21 / 16$}

\section{Harmonic Analysis}

The presence of the two families of forced harmonics is a well established feature in all systems to date that have measurements while in CT-induced motion ${ }^{4}$. Furthermore, the relationship between the rollout speed and the frequencies of these harmonics has been established for several years ${ }^{1}$. Therefore, another possible approach to generating a rollout forcing function is to impose this known harmonic content as a constraint. The approach used to define a set of basis functions containing sine and cosine functions at the known frequency as defined by the rollout speed. These basis functions were fit to the measured accelerometer data and transferred to the forcing function via analytical tools including the FEM of the CT and individual truck as well as other measurements sensitive to imposed forces (speed, JEL pressure, guide tube strain, truck acceleration). Equation 4 depicts the approximation of an acceleration time history as a summation of these harmonic basis functions.

$$
A(t)=\sum_{i=1}^{n h} S_{i} \sin \left(2 \pi f_{i} * t\right)+C_{i} \cos \left(2 \pi f_{i} * t\right) ;
$$

$S_{i}=$ sine coefficients for harmonic $i$,

$C_{i}=$ cosine coefficients for harmonic $i$,

$f_{i}=$ frequency of $i$ th harmonic $=i *$ scale $*$ speed 0 ,

$t=$ time in seconds,

scale $=17.6 *$ shoe spacing or $17.6 *$ roller spacing,

speed $0=$ mean speed of $\mathrm{CT}$, and

$n h=$ total number of harmonic basis functions.

Figure 13 shows and example of using this harmonic basis function approach to reconstruct an accelerometer time history from a verticle sensor on a truck below the JEL while the CT is unloaded and in motion at $7 \mathrm{mph}$. By taking account of the measured speed drift and dither of the system while fitting the data in increments, a high-fidelity fit of the entire time history is possible. Figure 13 shows the reconstruction and the data overlapping in the top plot. The middle plot shows the first 128 samples of data. Thre original and reconstructed traces are still largely indistinguishable. The bottom plot shows the requency-domain version of the data in the top plot. Note that the synthesized data (blue) is chosen to only retain harmonic content up to $30 \mathrm{~Hz}$. Also notice that much of the "noise" in the system is effectively described via tracking and reproducing the measured speed variations.

Figure 14 shows the magnitude of the sine (top plot) and cosine (bottom plot) parameters in the left hand side (while facing the page) pair. The right hand side data shows the same parameters converted to amplitude (top plot) and phase (bottom plot). The amplitude plot (right hand side top) is especially informative as the relative size of the parameters fit to the data is readily seen. Also note that this data is normalized (phase and amplitude scaled from average values) to allow better visualization of the data. This harmonic analysis approach has been found most useful as a supporting technique to analyze and transform data in support of other techniques mentioned herein. One example of this was an exercise to use a a static FEM of a truck to transfer this harmonic representation of the measured acceleration to displacements at the rollers as estimated of displacement forcing functions. Figure 15 shows such an approximate verticle displacement time history as the middle roller of an inboard track. This example had displacements that are approximately 5 times larger than expected. This error could be due to two likely issues: a static rather than dynamic FEM to relate roller dynamics to meansured acceleration; and the presence of modal content in the data that would be much reduced in actual forcing functions.

\section{Force and Frequency Response Function}

Another use of the harmonic analysis approach is to estimate a force time history acting on each truck by filtering measured data for the assumed harmonic content. A simplified assumption to exercise this approach is to assume that the measured accelerations closest to the truck/ground interface are largely driven by forced harmonics not structural dynamics. There is also a reuirement to assume the mass that is being moved by the acceleration. To begin with an estimate of the global speed of the CT is estimated from the speed sensor or from the harmonic content of the accelerometer signal. Figure 16 shows such an estimate in the top plot using a moving average of a measured speed signal from a $.7 \mathrm{mph}$ roll of the $\mathrm{CT}$. The middle plot contains a comparison to the original measured data. The bottom plot shows a comparison in the frequency domain. This estimated mean spead is then used to drive the harmonic fit of equation (4) to any of several measured data sets that might be highly enriched in forced data. Such data sets include the differential speed measurement, the pressure on the JEL system, the guide tube strains, or the truck accelerations closest to the ground. In this manner the signal can be enhanced in the harmonic content expected in the sensor signal as shown in the top plot and middle plot of Figure 17.

American Institute of Aeronautics and Astronautics 

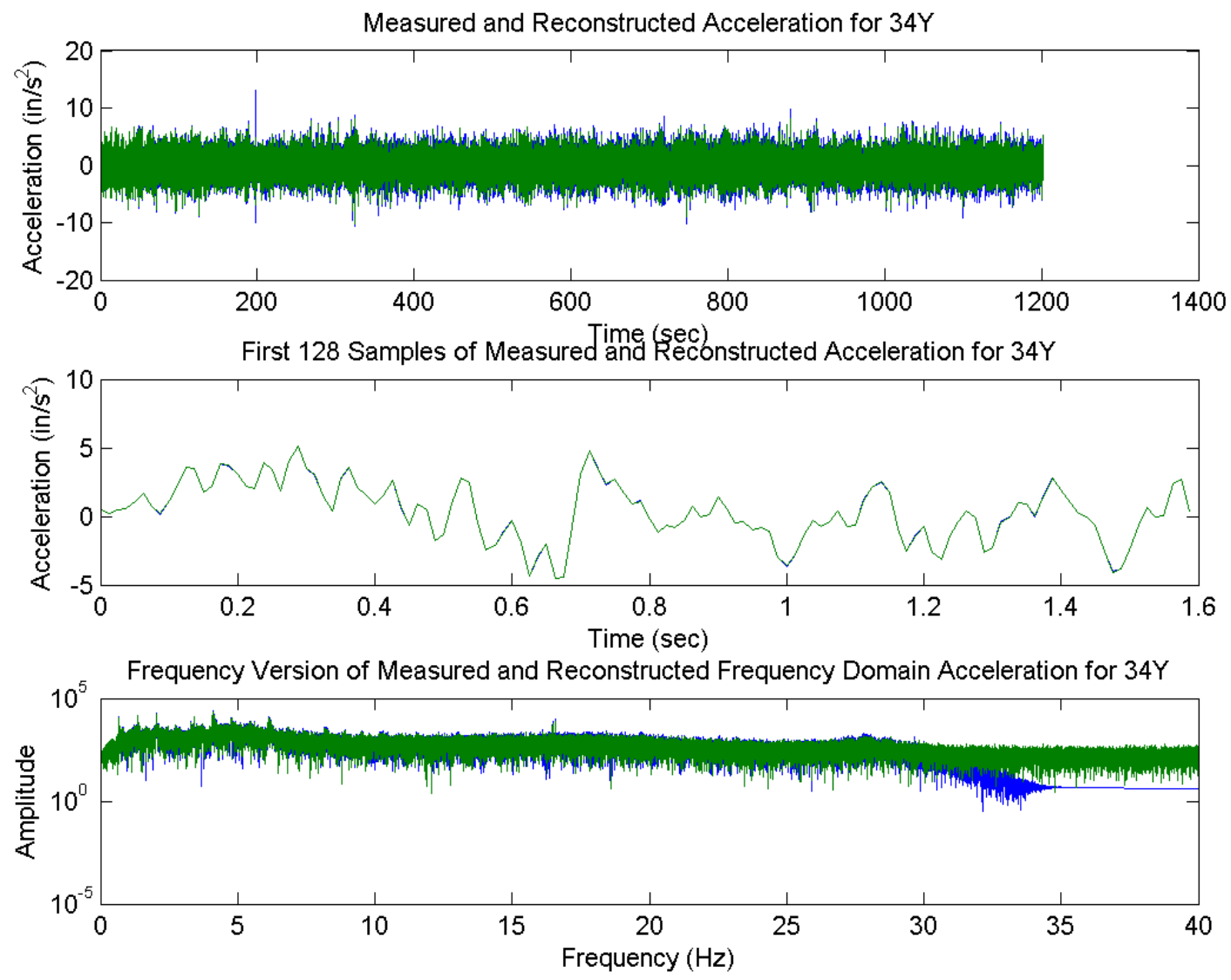

Figure 13. Reconstruction of Vertical CT Accelerometer Trace Using Harmonic Basis Functions
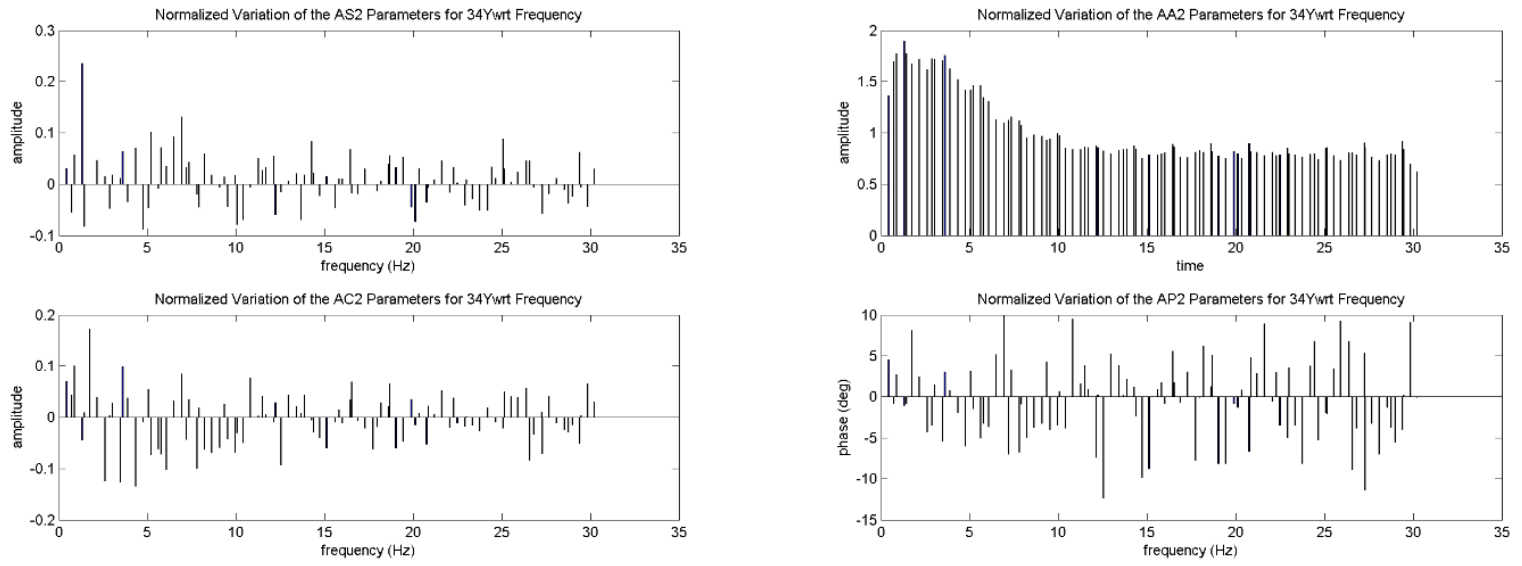

Figure 14. Sine and Cosine Parameters (LHS) and Converted to Amplitude and Phase (RHS) 

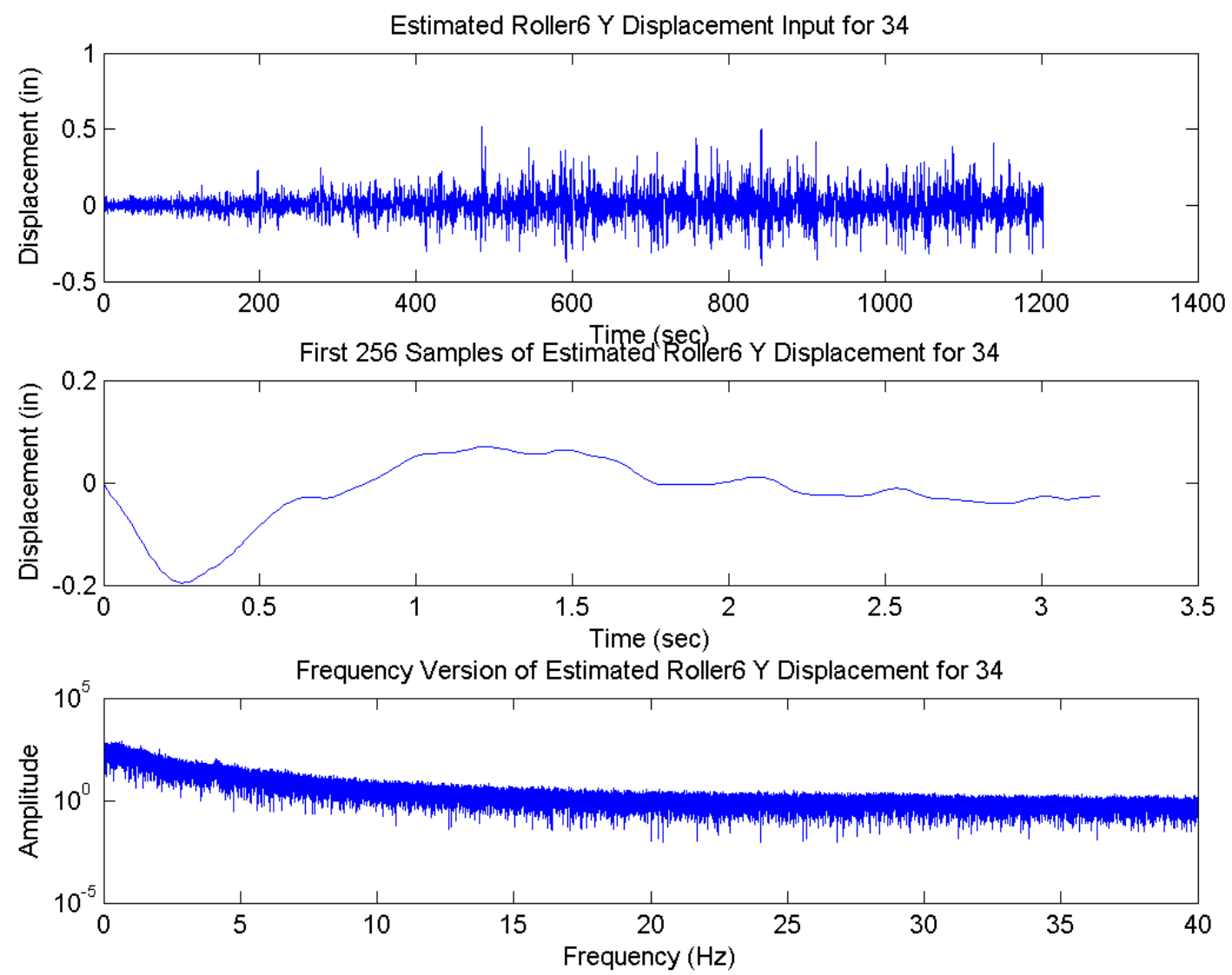

Figure 15. Roller Displacement Estimated from Acceleration Harmonic Basis Functions

For the example provided here, the harmonic filtered accelerometer signal is then multiplied by the mass of a truck to provide a first order approximation of the force trace acting on the entire truck in the measured direction. This estimate is provided in Figure 18. The top plot contains the full time record of estimated force (assuming the entire truck is being accelerated). The middle plot contains a zoomed version of this trace and the bottom plot the frequency domain version of the data. This force estimate as well as the first order forces in the other two directions are combined to the same three estimated forces at the the other three trucks to provide 12 force time histories to estimate Frequency Response Functions (FRFs). FRFs are a common mathematical respresentation of the dynamics of a system when subjected to input and output measurements. The input is a below JEL system vertical acceleration measurement, while the output is a vertical measurement above the JEL system. Figure 19 provides two estimates of the FRF (middle and bottom plot) that are in theory suppressed in forced content and enhanced in sturctual dyanamics. The top plot is an estimate of coherence, which shows how the two measurements appear to be related to each other.

FRFs hold great promise to allow an assessment of the underlying structural dynamics of a system, which would help address the problems being addressed by this effort. One advantage is that the FRF can be estimated by averaging across the length of the time histories of acquired data as well as across different operating speeds of the same vehicle/platform configuration. Another advantage is that the FRF can be used to implement frequency domain inverse techniques to estimate forcing functions ${ }^{15}$. Such generalized force estimation techniques can be used to constrain the forcing function to drive the system at selected or known locations. And finally, the FRF has the potential to separate the structural dynamics from the forced dynamics. The disadvantage of such a frequency domain approach is that an estimate of the input forcing function is needed to generate the resulting FRF. As such, continuing efforts in force estimation techniques such as SWAT or Inverse Structural Filter are suggested ${ }^{1,2,3,15}$. Alternatively, the forcing functions may need to be defined at locations where measurements exist, which can corrupt the input estimates with system effects and make generalization other systems more difficult.

American Institute of Aeronautics and Astronautics 

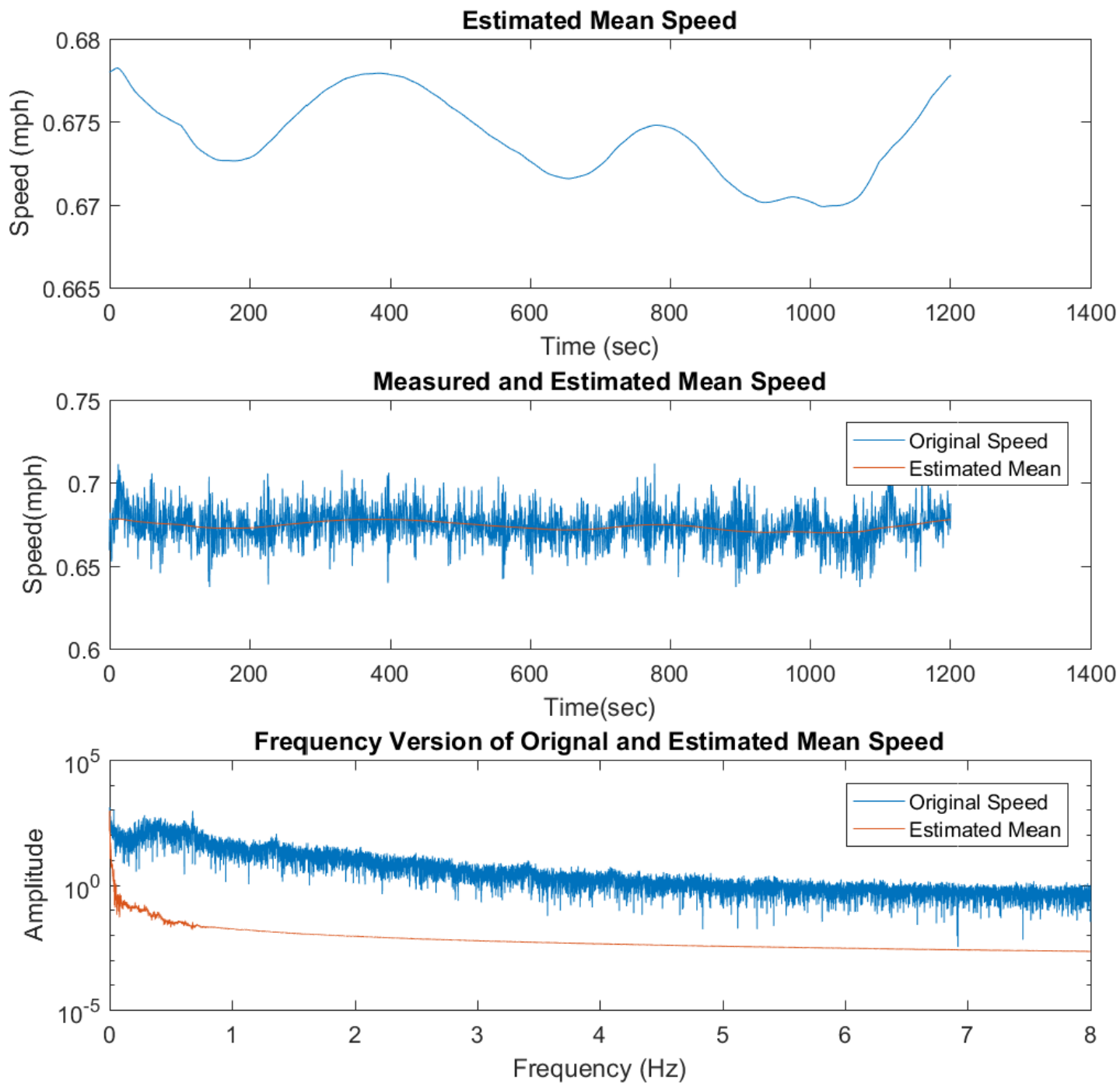

Figure 16. Estimated Mean Speed From Moving Average Fit of Measured Speed

\section{Multi-Body Dynamics Analysis}

The most refined analysis work currently underway involves the multi-body dynamics analysis work using the ADAMS software. This work is specifically targeting understanding and mechanizing the physics associated with the ground/shoe/track/roller interactions ${ }^{10}$. The current state of this analysis includes a single truck with two tracks of 11 rollers each has been modelled. This model can treat the truck as a flexible body or as a rigid body. The ability to output accelerations as sensor locations is implemented. Also concentrated mass to cover the effects of the CT chassis and any launch platform/launch vehicle payload can be added. Work is underway to add a fully looped track, articulating tracks, and ground deflection effects to the model. The system can be driven with realistic measurementbased speed. In addition to outputs corresponding to the measured outputs, roller axle displacements, and changing forces responding to variable roller/shoe interactions can be output as well. The displacement and force outputs can be subjected to harmonic basis function analysis to allow expansion in time and operations. 

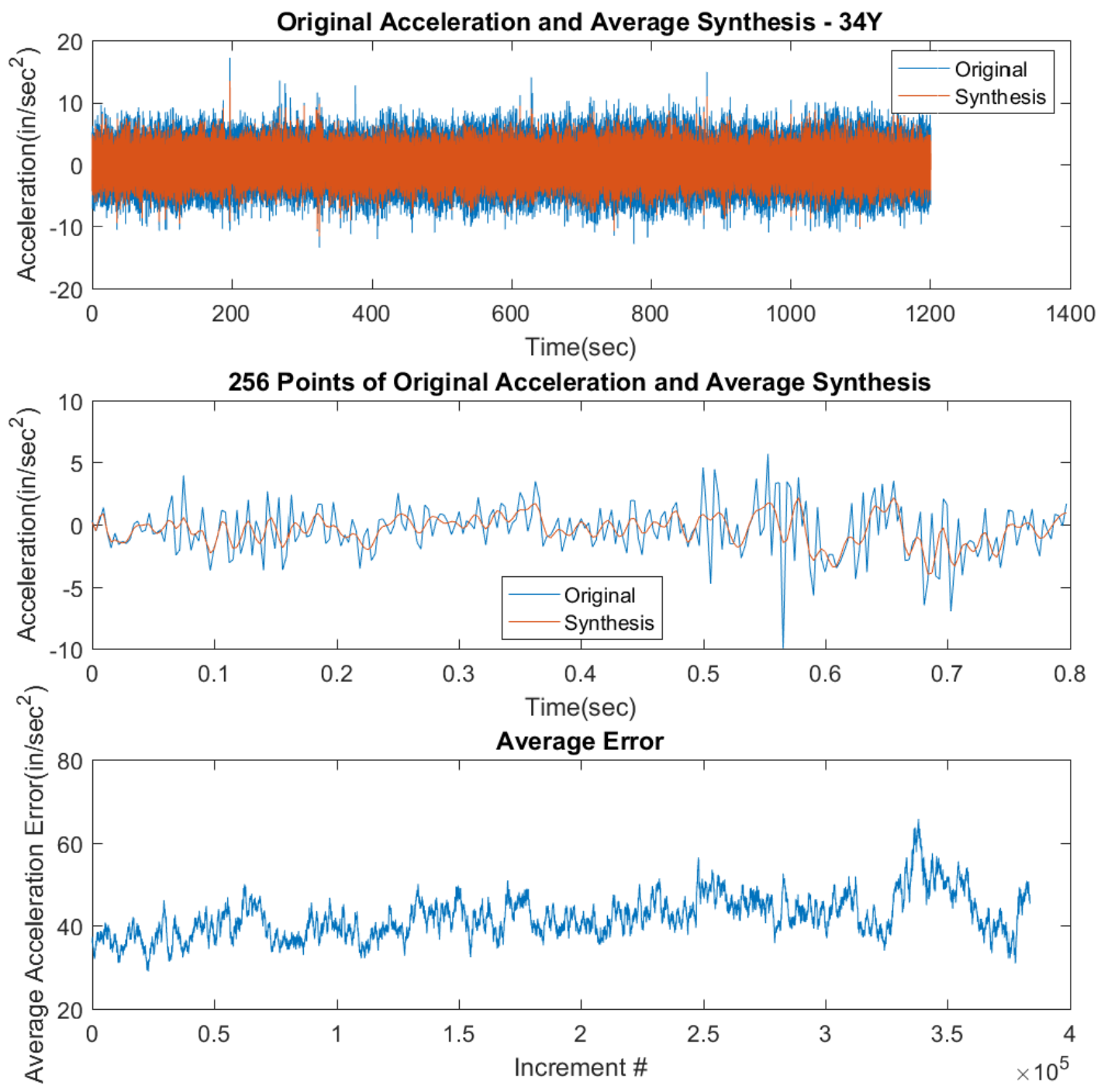

Figure 17. Measured Acceleration And A Harmonic Fit to $60 \mathrm{~Hz}$

Figure 20 shows a recent multi-body dynamics model of a single CT truck. Note that this image of the model uses a flat track. The physical scars that an actual truck possess for the guide tube and JEL cynlinders (large circular depression on top surrounded by four smaller circular depressions) as well as the gear system and track sprockets (cutouts at either end) are seen on the model. The 11 rollers are also seen on the outboard track. Advanced versions of this model are in development that add the track sprockets at either end and change the track to a continuous loop over the truck as seen in the real hardware. Refer to Figures 3, 4, and 5 for additional context with the actual structure. Figure 21 shows a 90 second run of the Adams model (top plot LHS) and a three second zoom (middle plot LHS). The bottom plot also contains the same data but in the frequency domain. All the plots also show the analytical fit using 21 harmonics up to six $\mathrm{Hz}$. Once this fit is made, then the entire data set can be approximately extended to the 1200 second extent of the corresponding measured data as shown in the three RHS figures. Note that there appears to be more complicated dynamics in the original time record due to the peaks not covered by the assumed harmonics or the chosen frequency cut-off. Longer time histories, different model damping, hardware observations, and refined signal processing are being used to study these effects. The 1200 second expanded frequency data from the bottom plot of the RHS shows that the shoe spacing frequencies were captured well but the roller spacing harmonics are still suppressed.

American Institute of Aeronautics and Astronautics 

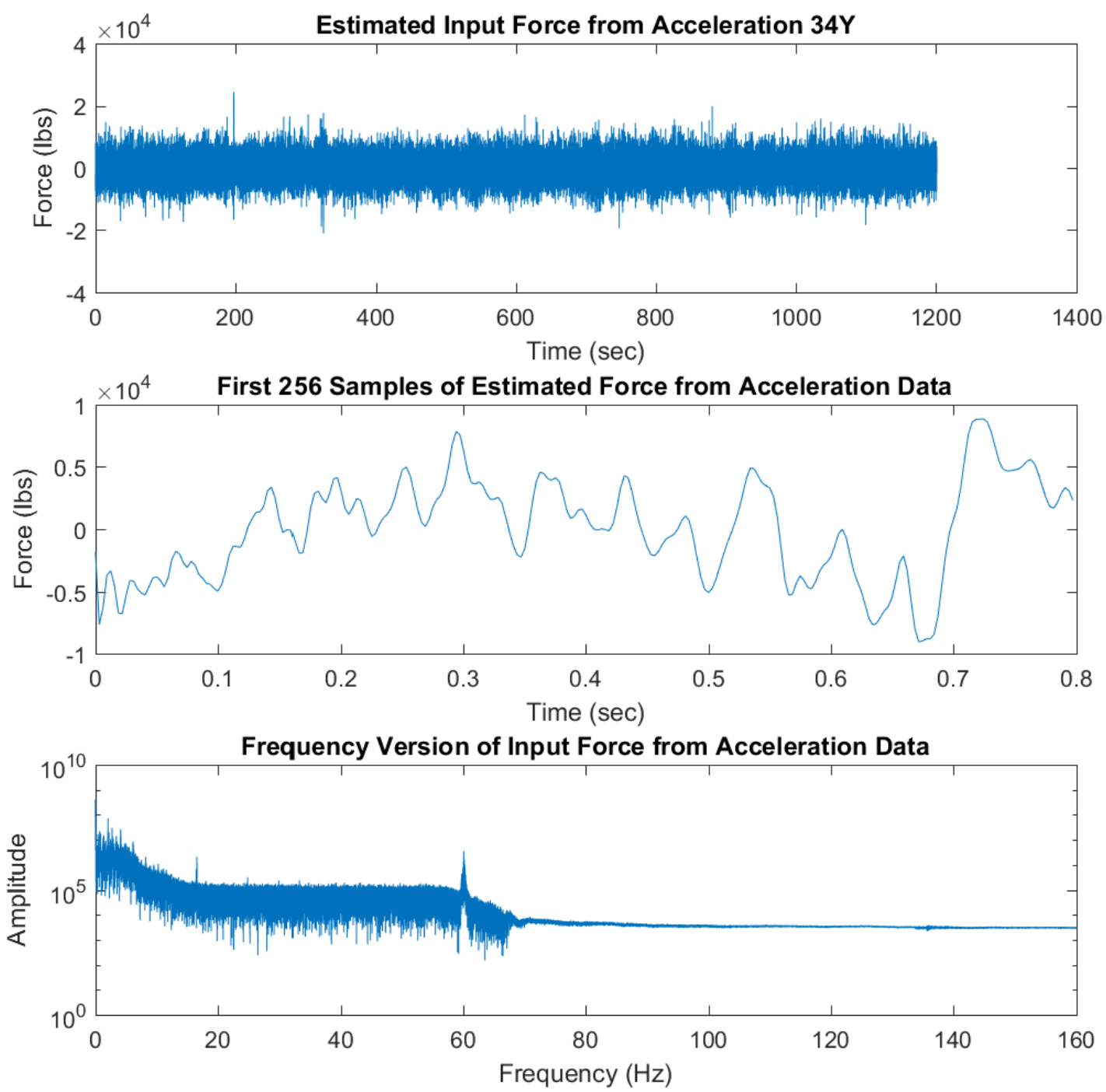

Figure 18. First Order Estimate of Vertical Force Generated by One Truck during .7 MPH Speed

Figure 22 shows a set of results from driving an FEM of the CT with a harmonically expanded set of Adams data. This data shows that there was some reasonable coverage in the fore-aft $(\mathrm{X})$ direction and the side-to-side $(\mathrm{Z})$ direction. However, the up-down (Y) direction was over predicted. Part of the issue is that an estimate of phase and local speed differences between the four truck needs to be taken into account in a more realistic fashion. It cannot be assumed (as was used in this data run) that all eight tracks were completely synchronized. Another issue is that Adams runs need longer time estimates to reduce boundary and signal processing issues. Finally the harmonic fits are likely affected by using speed variations containing noise. Hence, filtering of the speed data before driving the harmonic analysis is in order. Also subsequent work has suggested that the flexible body effects of the truck would enhance the Adams predictions. Unfortunantly, flexible body models significantly increase the Adams run times. 

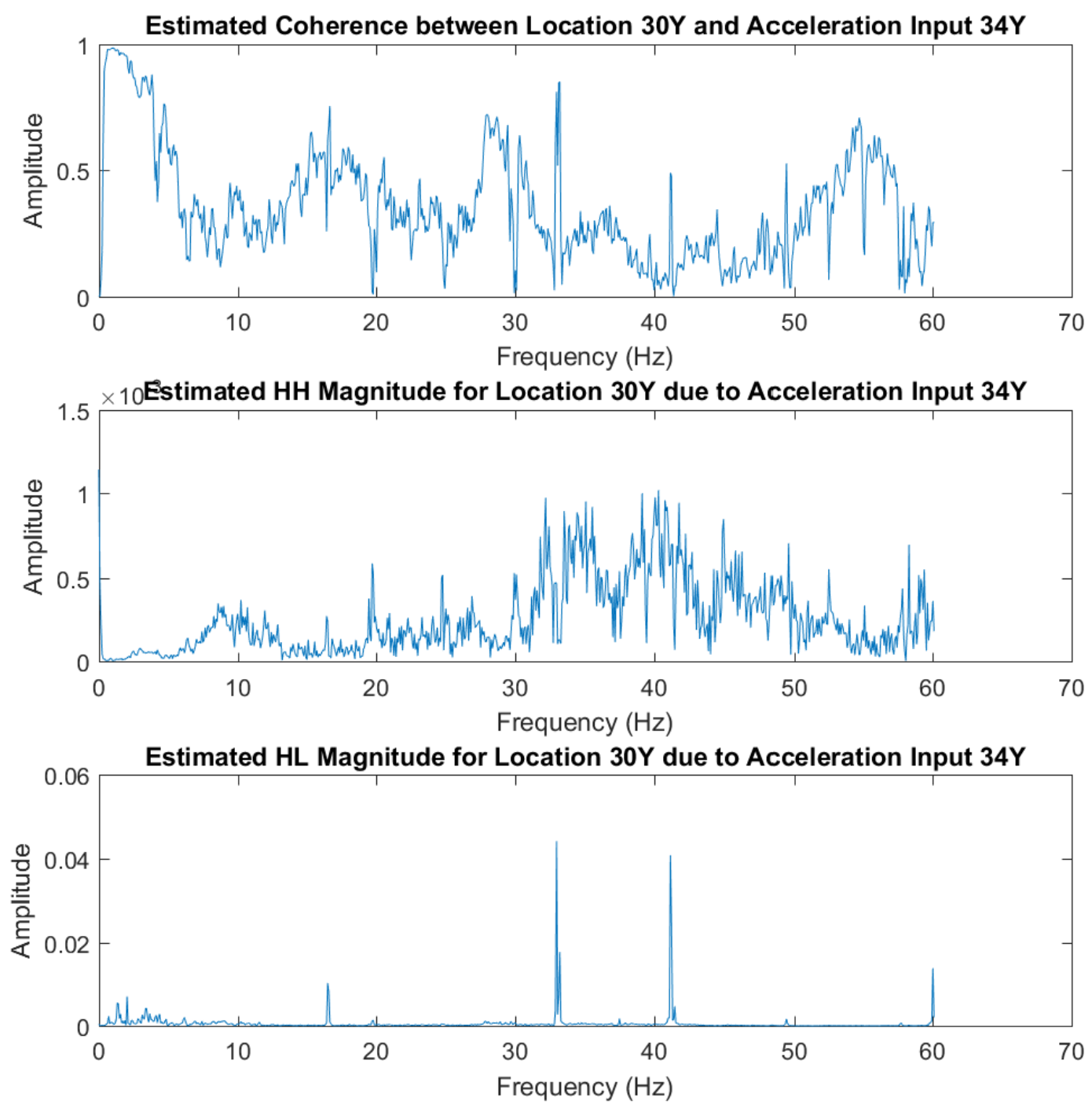

Figure 19. Coherence and High and Low Estimates of Frequency Response Functions

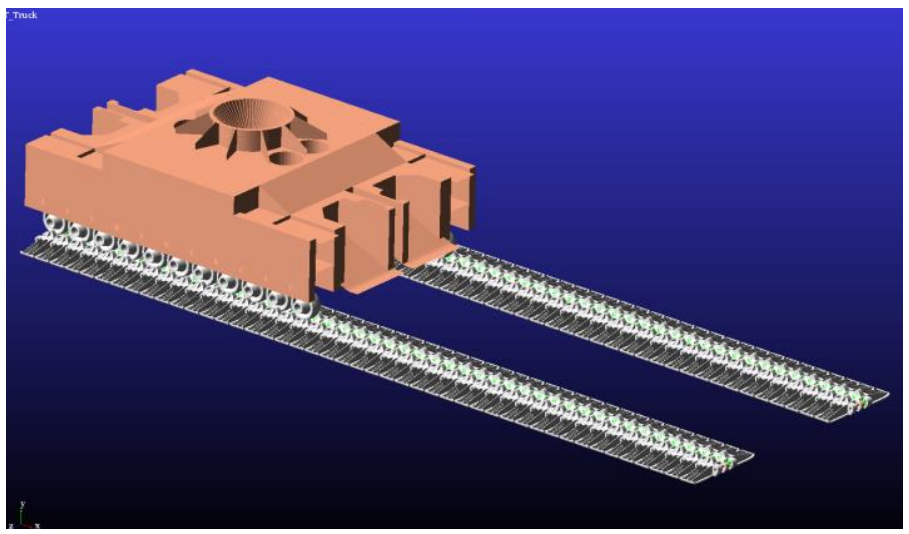

Figure 20. Multi-Body Dynamics Model of a Single Truck 

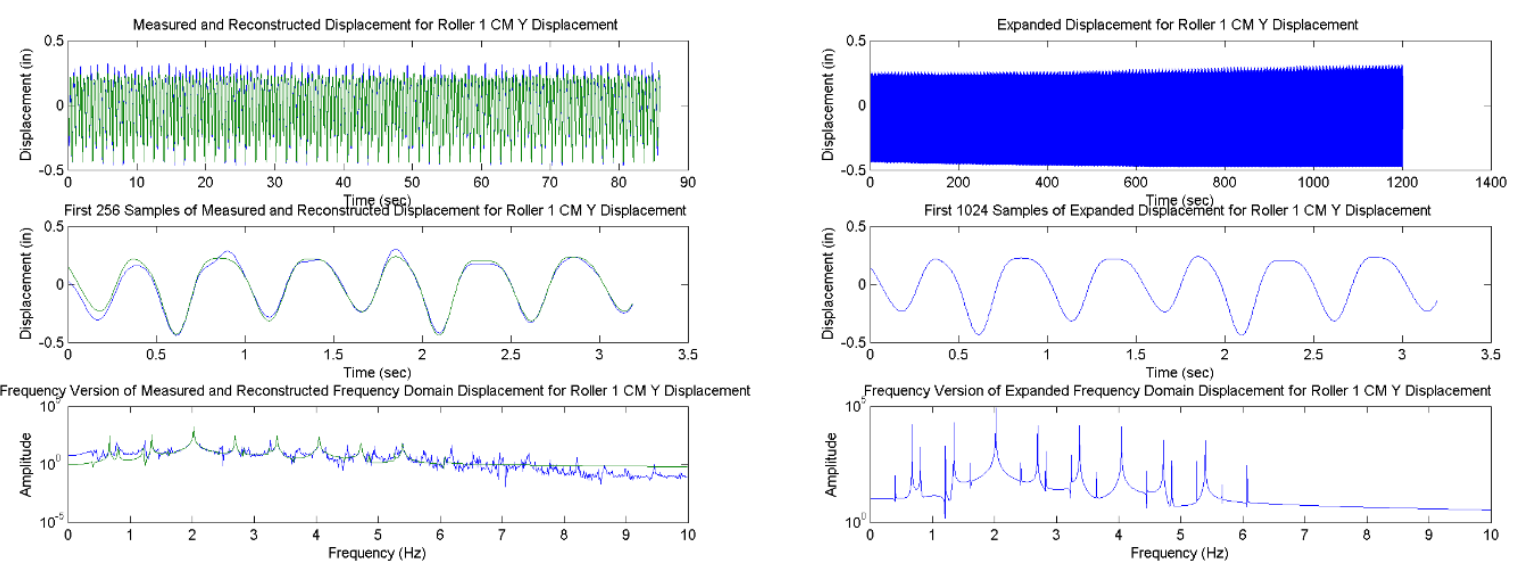

Figure 21. Multi-Body Dynamics Results From a Single Truck and Expanded Using Harmonic Fit
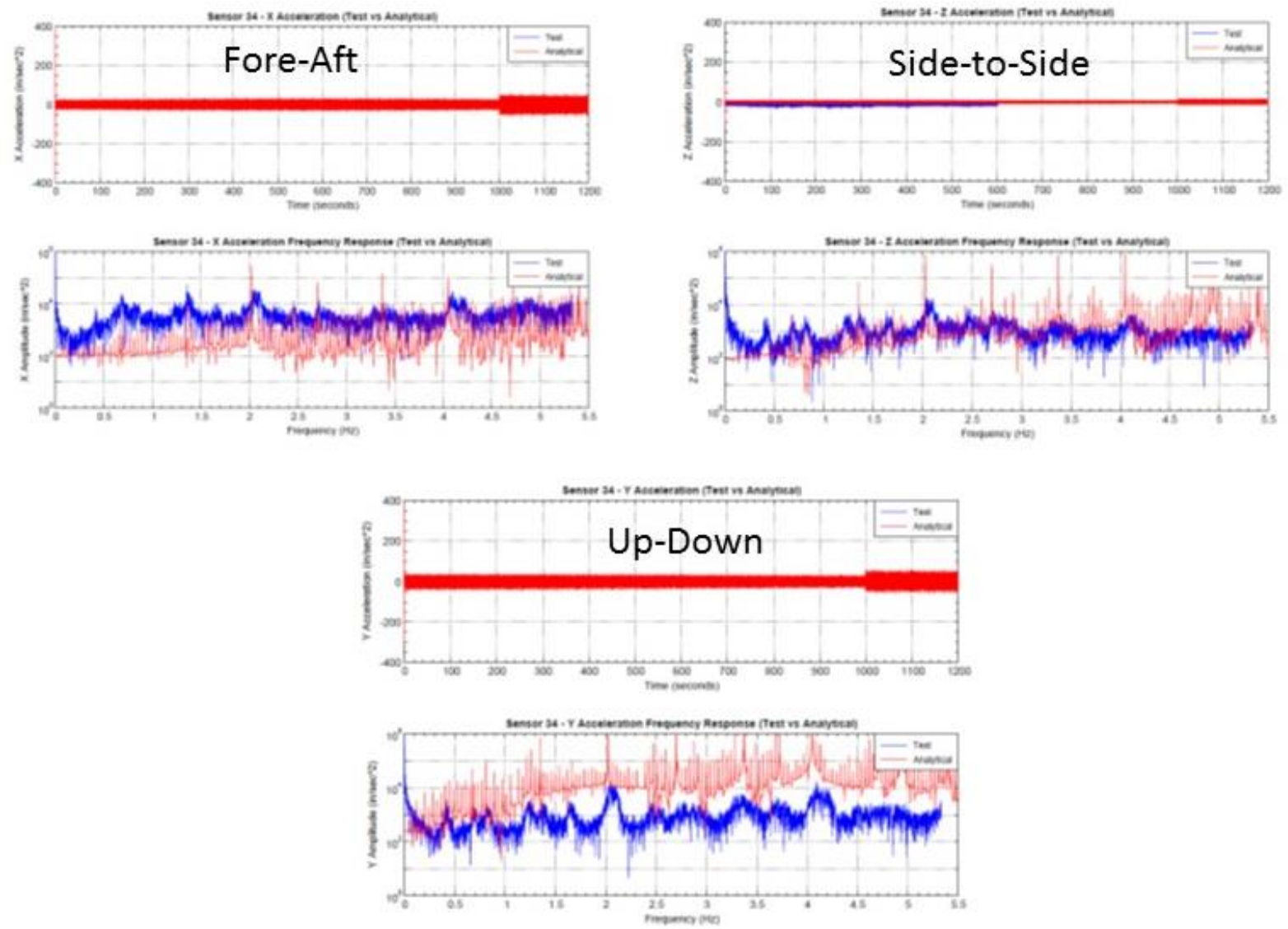

Figure 22. FEM Results From a Model Driven With Harmonically-Expanded Adams Data

\section{Problem \#1 - Forcing Function for FEM Analysis}

As mentioned earlier, the first problem concerns the production of a forcing function to drive FEM models enabling Coupled Loads Analyses (CLAs). These CLAs predict combined effects on the launch vehicle and launch platform during rollout loading. Past analyses have used enforced acceleration loads at the interface between the CT and the launch platform. The CT is not even modelled in this approach and any CT dynamics are part of the forcing function (and potentially dynamic effects of the payload). Obviously, this approach would be quite simple to implement if measured acceleration data were available from a very similar system under roll at the same speeds as the CLA plans

American Institute of Aeronautics and Astronautics 


\section{$11 / 21 / 16$}

call out. However, since the system to be modelled (SLS with SLS-ML on SLS-era CT) has not been assembled and will not be assembled or tested until seaveral months after the CLA results are needed, the trivial solution is not viable. Hence, the analytical tools and measured data mentioned in the previous section need to be used to develop a plan for contructing such a forcing function to assess and if needed replace the current forcing function. This plan needs to include three parts: (A) forcing function development plan, (B) forcing function verification plan, and (C) forcing function backup plan. Each of these three aspects will be discussed based on the current state-of-knowledge.

\section{Forcing Function Development Plan}

The targeted forcing function will be acceleration inputs to the launch platform at the interface to the CT. Since the vehicle and the launch platform will not have been tested by the delivery of the forcing function, two forcing functions will be produced: the final set which is the deliverable but a product of non-validated models and an intermediate set that is derivable from available data. The final forcing functions will necessarily be derived from an analytical model of the SLS and the SLS-ML and verified with a combination of the most similar data available coupled with analytical results using a separate and different modelling approach. Since the driven system model (SLS + SLS-ML) can be assumed to exist, the issue will be to provide the plan for developing the intermediate interface acceleration estimates. These estimates will come from an analytical FEM system that includes the SLS, the SLS-ML, and the CT and driven by inputs that are as unaffected by system dynamics as possible (i.e. as close to the ground/shoe/roller interfaces as possible). Given the current data, these intermediate forcing functions will likely be forces acting below the JEL system on each of the four trucks of the CT as this is the location of the data closest to the desired interfaces. Enforced accelerations in lieu of forces are a possible modification to this plan (if needed) that will not negate the plan. The intermediate forces will be derived from measured/estimated accelerations from the trucks and augmented with forces from JEL system pressures. Low-pass filtered/smoothed speed measurements will also be used. These measured accelerations will be derived from all of the data acquired to-date on Shuttle-era hardware. A most critical database will be the rollout data from the CxP ML on the Shuttle-era CT. The CxP ML data is critical because it is a transitional dataset between the Shuttle MLP and the SLS ML. The current plan is for the estimated forces to be time-shifted to produce data at each desired rollout speed as was performed in reference ${ }^{5}$.

The conversion of below-JEL accelerations to the intermediate forces in the previous section is the critical aspect of this planned process. This conversion will begin by estimating the mass needed to scale the accelerations of measured below the JEL system by mass values that are derived to match the axial forces estimated from JEL system pressures. Transverse and fore-aft directions will use the same scaling. Although it is possible that transverse and fore-aft scaling will be affected by guide tube strains measured in the SLS-CT and SLS-ML rollout tests. Using these scaled versions of the below-JEL accelerations as inputs and the accelerations measured elsewhere as outputs, average FRF and inverse FRF transfer functions will be calculated. These will be used to estimate input forces acting at the below-JEL accelerometer measurements using a frequency domain inverse calculation ${ }^{15}$. These estimated forces will be filtered using the harmonic basis functions appropriate for each measured speed. During the development phase, FEM models of the Shuttle-era data sets will be used to verify the forcing functions/transfer functions/mass scaling aspects of this plan. This verification will be performed by matching enforced FEM analysis to measured data from the rollout databases mentioned previously. It is possible that the SWAT approach or the Inverse Structural Filter approach can replace the frequency domain inversion approach if verification suggests such an action ${ }^{1,3,5,15}$. It should be noted that a critical input to thie process will be a model of the SLS-era CT, which is still in production. This model will be validated via the same FRF transfer function calculation mentioned above. Using FRF's derived from the CT only tests, the modal content of the CT will be measured and matched via model correlation.

\section{Forcing Function Verification Plan}

The final forcing functions will be verified first by assessing the intermediate forces via an Adams model of a simgle truck propelled by measured speed variations properly loaded with payload mass. Harmonic analyses will be used to expand the Adams data to longer times and estimate the effects of other trucks. Direct comparison to measured accelerations and analytical estimates of whole truck forces from the Adams analyses will be used to compare to the intermediate forcing functions listed above. It should be noted that validation will occur post-delivery when a validated SLS-ML model and data from the SLS are available. Second, the final forcing functions will also be compared to measured accelerations from the previously mentioned existing data sets. In additional to a direct comparison of the acceleration values, output accelerations from driving the SLS model with measured acceleration time histories will contribute to verification. It should be noted, that it is possible that other measured accelerations may prove conservative with respect to the final forcing functions as system dynamics may be artificially increasing response.

American Institute of Aeronautics and Astronautics 


\section{$11 / 21 / 16$}

\section{Forcing Function Backup Plan}

A backup to the development and verification plans mentioned above is required as there are technical and schedule uncertainties that might call for an alternative. The backup for the final forcing functions will be to directly use measured CT/launch platform accelerations. Again special emphasis will be given to the CxP-ML data. It should be noted that the final forcing functions will enveloped from multiple measured sources in this case, they will contain conservatism in the resulting loads that may not be desired late in the design.

\section{Problem \#2 - Forcing Function for Test Analysis}

The second problem to be solved involves estimating the forcing function directly from the DRT test of the SLS vehicle on the SLS ML. This needs to be performed in a short period of time and to allow the generation of data that can be used to directly assess and correlate the model of the launch vehicle. The development plan, validation plan, and backup plan will again be provided.

\section{Forcing Function Development Plan}

The previous section covered the estimation of FRFs from estimated inputs (based on measurements) to measured outputs. This approach is very similar to traditional approaches to perform model validation based on traiditional modal testing. Also, due to the ability to average long records of data and potentially operate completely on meansured data are advantages to this approach. Hence, measured data from the below-JEL truck acceleration, forces estimated form the JEL system pressures, and forces estimated from guide tube strains are expected to cover a full range of inputs to the system and will be used as inputs to the FRF, inverse FRF, and force estimation processes. As before, the current plan is to use a frequency domain inversion process to place the force inputs where needed. It is expected that any need to transition to other force estimation processes (or input accelerations) will be determined and implemented using the rollout testing of the SLS-ML and will not be an issue when processing DRT data. The resulting FRF's and inverse FRF's will again be processed using traditional approaches The FRFs will be used to extract modal properites of the coupled sytem. The most uncertain aspect of this approach is still the selection of weighting for the below truck accelerations. However, the process to derive intermediate forcing function scaling from Problem \#1 above will be available for use and will be enhanced by having validated CT and ML models.

\section{Forcing Function Verification Plan}

The complete process for deriving forces and FRFs will be exercised and validated during the SLS-ML roll to the pad. In fact, the resulting FRFs will also be available for use in correlating the ML model. The validation of the actual forces used to develop FRFs will be validated via Adams runs of a single truck using measured speed inputs with measured forces from JEL system and guide tubes.

\section{Forcing Function Backup Plan}

The backup to develop the test-based forcing functions will be to drive the DRT data using operational operational modal analysis tools ${ }^{16,17}$. This approach has been exercised on Shuttle ${ }^{6}$ and Ares1-X rollout data with varying degrees of success. As with the primary approach, this backup approach will be completely based on measured data. However, there are limitations to the parameter space that can be extracted using OMA processing. Specifically, closely spaced modes and modal parcipation scaling would not be readily extracted.

\section{Conclusions}

The SLS rollout environment to the launch pad will be rich with forced harmonics and higher order effects. This situation can create design and fatigue loads, especially when the forcing functions interact with the system dynamics. However, the same environment can be a useful operational scenario for extracting structural dynamics information. The previous, ongoing, and future work in this area create a need to integrate a broad range of data and understanding. This paper represents the first phase of this integration by providing an overview of the hardware, the definitions, the existing and planned data, and previous and ongoing analyses. This information provided is necessary to allow the needed assimilation and integration. This paper has also initialized the integration by providing the primary, validation, and backup plans for solving two problems: (1) developing a set of rollout forcing functions (as enforced displacements) for enabling Finite Element Model (FEM) Coupled Loads Analyses (CLAs); and (2) deriving forcing functions (as input forces) and Frequency Response Function (FRFs) with respect to the measured outputs during the rollout of the Space Launch System (SLS) and the Mobile Launcher (ML). 


\section{$11 / 21 / 16$}

A critical aspect of the current plans for addressing both problems will be to use a frequency domain inversion process to estimate forces. The required transfer functions are planned to be developed using measured accelerations closest to the interaction between the ground, the tracks, and the trucks of the Crawler Transporter (CT). These accelerations will be mass scaled using information measured pressures in the hydraulic systems supporting the payload. A harmonic curve fit will be performed to filter the forcing functions based on the known geometries of the hardware and the measured speeds. The plans include utilizing measurement-only estimations of force and transfer functions but verified with advanced multi-body dynamics and/or data form similar systems. Backup plans using alternative force estimation techniques, hybrid analytical/test methods, and operational modal analysis techniques are provided. The integration and plans provided herein are currently beign subjected to the assessments provided by the databases in place from NASA's previous Shuttle and Constellation Programs. The culmination of the integration, implementation, and validation of this work will be the subject of subsequent publications and reports.

\section{References}

${ }^{1}$ G. James, T. Carne, and B. Wilson., "Reconstruction of the Space Shuttle Roll-Out Forcing Function", Proceedings of the 25 $5^{\text {th }}$ International Modal Analysis Conference, Orlando, FL, February 19-23, 2007.

${ }^{2}$ T. Carne, V. Bateman, and R. Mayes, "Force Reconstruction Using a Sum of Weighted Accelerations Technique", Proceedings of the 10 $0^{\text {th }}$ International Modal Analysis Conference, San Diego, CA, Feb. 3-7, 1992.

${ }^{3} \mathrm{M}$. Allen and T. Carne, "Comparison of Inverse Structural Filter (ISF) and Sum of Weighted Accelerations Technique (SWAT) Time Domain Force Identification Mehtods", Proceedings of the 47 th AIAA/ASME/ASCE/AHS/ASC Structures, Structural Dynamics, and Materials Conference, Newport, RI, May 1-4, 2006.

${ }^{4}$ K. Elliott, R. Buehle, and G. James, "Space Shuttle Transportation (Rollout) Loads Diagnostics", Proceedings of the $23^{\text {th }}$ International Modal Analysis Conference, Orlando, FL, Jan. 31 - Feb. 3, 2005.

${ }^{5}$ G. James, T. Carne, K. Elliott, and B. Wilson, "Estimation of the Space Shuttle Roll-Out Forcing Function", Proceedings of the $23^{\text {th }}$ International Modal Analysis Conference, Orlando, FL, Jan. 31 - Feb. 3, 2005.

${ }^{6}$ R. Buehle and K. Kappus, "Operating Deflection Shapes for the Space Shuttle Partial Stack Rollout", Proceedings of the $23^{\text {th }}$ International Modal Analysis Conference, Orlando, FL, Jan. 31 - Feb. 3, 2005.

${ }^{7}$ J. Townsend, I. Torres, and K. Smalley, "Space Shuttle Base Drive Dynamic Analysis for Derivation of Load Fatigue Spectra", Proceedings of the $23^{\text {th }}$ International Modal Analysis Conference, Orlando, FL, Jan. 31 - Feb. 3, 2005.

${ }^{8}$ S. Del Basso, J. Dolenz, and L. Wilson, "Space Shuttle Partial Stack Rollout Test Analytical Correlation in Support of Fatigue Load Development", Proceedings of the 23th International Modal Analysis Conference, Orlando, FL, Jan. 31 - Feb. 3, 2005.

${ }^{9} \mathrm{M}$. Chamberlain and S. Hahn, "Reconstruction of Ares I-X Integrated Vehicle Rollout Loads", Proceedings of the 52 AIAA/ASME/ASCE/AHS/ASC Structures, Structural Dynamics, and Materials Conference, Denver, CO, April 4-7, 2011.

${ }^{10}$ B.C.Chen, G. Ortiz, and C.-Y. Peng, "Modelling of Crawler Transporter for Prediction of Rollout Forcing Functions", Proceedings of the 2015 Spacecraft and Launch Vehicle Dynamic Environments Workshop, El Segundo, CA, June 2-4, 2015.

${ }^{11}$ D. Lavrinc, "Have a Rocket to Launch? NASA's Massive Launch Platforms are Now on Sale", Wired, August 23, 2013, https://www.wired.com/2013/08/mobile-launcher-platform.

${ }^{12}$ C. Bergin, "Mobile Launcher Redesign Plan for SLS Nears Completion”, NASA Spaceflight.com, Februrary 5, 2013, https://www.nasaspaceflight.com/2013/02/mobile-launcher-redesign-plan-sls-completion.

${ }^{13}$ L. Herridge, "Mobile Launcher Mods Will Support NASA's Next-Generation Rocket and Spacecraft", NASA Kennedy Space Center, FL, August 21, 2015, http://www.nasa.gov/feature/mobile-launcher-mods-will-support-nasas-next-generation-rocket-andspacecraft.

${ }^{14}$ H. Vold, "Final Report on Model-based Continuum Interpolation of Measured Vector Time Histories Through SpatioTemporal Filtering”, ATA Project \#54047, ATA Engineering Inc., San Diego, CA, June 18, 2006.

${ }^{15} \mathrm{M}$. Allen and T. Carne, "Delayed, Multi-step Inverse Structural Filter for Robust Force Identification", Mechanical Systems and Signal Processing, July 2008, pp. 1036-1054.

${ }^{16}$ G.H. James, T.G. Carne, and J.P. Lauffer, "The Natural Excitation Technique (NExT) for Modal Parameter Extraction from Operating Structures," SEM International Journal of Analytical and Experimental Modal Analysis, Vol. 10, No. 4, October 1995.

${ }^{17}$ T. G. Carne and G. H. James, "The Inception of OMA in the Modal Testing Technology for Wind Turbines", Mechanical Systems and Signal Processing, July 2010, pp. 1213-1226.

American Institute of Aeronautics and Astronautics 